\title{
Heating and Acceleration of the Fast Solar Wind by Alfvén Wave Turbulence
}

\author{
A. A. van Ballegooijen ${ }^{1}$, M. Asgari-Targhi ${ }^{1}$
}

\begin{abstract}
We present numerical simulations of reduced magnetohydrodynamic (RMHD) turbulence in a magnetic flux tube at the center of a polar coronal hole. The model for the background atmosphere is a solution of the momentum equation, and includes the effects of wave pressure on the solar wind outflow. Alfvén waves are launched at the coronal base, and reflect at various heights due to variations in Alfvén speed and outflow velocity. The turbulence is driven by nonlinear interactions between the counter-propagating Alfvén waves. Results are presented for two models of the background atmosphere. In the first model the plasma density and Alfvén speed vary smoothly with height, resulting in minimal wave reflections and low energy dissipation rates. We find that the dissipation rate is insufficient to maintain the temperature of the background atmosphere. The standard phenomenological formula for the dissipation rate significantly overestimates the rate derived from our RMHD simulations, and a revised formula is proposed. In the second model we introduce additional density variations along the flux tube with a correlation length of $0.04 R_{\odot}$ and with relative amplitude of $10 \%$. These density variations simulate the effects of compressive MHD waves on the Alfvén waves. We find that such variations significantly enhance the wave reflection and thereby the turbulent dissipation rates, producing enough heat to maintain the background atmosphere. We conclude that interactions between Alfvén- and compressive waves may play an important role in the turbulent heating of the fast solar wind.
\end{abstract}

Subject headings: Magnetohydrodynamics (MHD) - Sun: solar wind - Sun: corona - Sun: magnetic fields - turbulence - waves

\section{Introduction}

The fast solar wind has velocities in the range 500 to $800 \mathrm{~km} \mathrm{~s}^{-1}$, and originates in coronal holes, which are low-density, open magnetic field structures in the Sun's corona (see

\footnotetext{
${ }^{1}$ Harvard-Smithsonian Center for Astrophysics, 60 Garden Street, Cambridge, MA 02138, USA
} 
Zirker 1977). The fast wind is believed to be driven by Alfvén waves that are launched in the photosphere and propagate outward along the open field lines (e.g., Parker 1965; Heinemann \& Olbert 1980; Velli 1993; Dmitruk et al. 2002; Suzuki \& Inutsuka 2005, 2006; Cranmer et al. 2007; Verdini et al. 2009; Cranmer et al. 2015). Alternatively, the waves may be launched by micro-flares in the chromospheric network (e.g., Axford \& McKenzie 1992). Direct evidence for the existence of Alfvén waves comes from in situ observations of fluctuations in the solar wind (e.g., Coleman 1968; Belcher \& Davis 1971; Bale et al. 2005). The waves have a broad spectrum of wavenumbers and frequencies, and are believed to be turbulent, that is, the different wave modes interact nonlinearly with each other, and the wave energy is continually being transferred from larger to smaller spatial scales (e.g., Hollweg et al. 1982; Hollweg 1986; Matthaeus et al. 1990). In the inertial range the turbulence is highly anisotropic: the fluctuating field $\delta \mathbf{B}$ is nearly perpendicular to the background field $\mathbf{B}_{0}$, and varies more rapidly in the direction perpendicular to $\mathbf{B}_{0}$ than along it. Alfvén waves have also been detected by studying wave-like phenomena in the solar atmosphere (Tomczyk et al. 2007; Tomczyk \& McIntosh 2009; De Pontieu et al. 2007; Morton et al. 2015). These observations provide evidence for the existence of counter-propagating Alfvén waves in the corona. The Interface Region Imaging Spectrograph (IRIS) has observed signatures of Alfvén waves carried by network jets in the transition region of coronal holes (Tian et al. 2014), indicating that Alfvén waves are injected into the corona at the base.

The mechanisms by which the fast solar wind is heated and accelerated are not yet fully understood. Alfvén waves are the most promising wave type for transporting energy over large distances in the corona and solar wind (Barnes 1966; Velli et al. 1989; Matthaeus et al. 1999; Suzuki \& Inutsuka 2006; Chandran et al. 2011). However, it is not clear how the wave energy is transferred to the particles. Some authors have argued that the waves have very high frequencies $(\sim \mathrm{kHz})$ and interact with the particles via ion-cyclotron resonances (Tu \& Marsch 1995). Such models can explain why the different ion species have different temperatures and outflow velocities, but it is not clear how the ion-cyclotron waves would be produced. Others have suggested that the coupling between Alfvén- and compressive waves plays an important role in Alfvén-wave dissipation (Kudoh \& Shibata 1999; Moriyasu et al. 2004; Suzuki \& Inutsuka 2005, 2006; Matsumoto \& Shibata 2010).

Many authors have focused on wave turbulence as a mechanism for coronal heating (e.g., Hollweg 1986; Velli et al. 1989; Matthaeus et al. 1999). One likely source of turbulence is the nonlinear interaction between counter-propagating Alfvén waves (Iroshnikov 1963; Kraichnan 1965). The waves can be described in terms of Elsasser variables, $\mathbf{z}_{ \pm} \equiv \mathbf{v}_{1} \mp \mathbf{B}_{1} / \sqrt{4 \pi \rho_{0}}$, where $\mathbf{B}_{1}$ and $\mathbf{v}_{1}$ are the magnetic and velocity fluctuations of the waves, and $\rho_{0}$ is the mean plasma density. In a homogeneous medium the $\mathbf{z}_{+}$and $\mathbf{z}_{-}$fields would represent outward and inward-propagating waves, respectively. However, in an inhomogeneous atmosphere such as 
the corona the Alfvén speed $v_{A}(r)$ varies with radial distance $r$, and the $\mathbf{z}_{+}$and $\mathbf{z}_{-}$waves are linearly coupled to each other (Heinemann \& Olbert 1980; Velli 1993; Hollweg \& Isenberg 2007). Therefore, the $\mathbf{z}_{+}$waves launched by the Sun naturally produce $\mathbf{z}_{-}$waves at larger heights. Velli et al. (1989) have shown that in an inhomogeneous atmosphere with dominantly outward waves the $\mathbf{z}_{+}$field generates a "secondary component" in the $\mathbf{z}_{-}$field, which travels in the same direction as the $\mathbf{z}_{+}$primary field. Therefore, in general the $\mathbf{z}_{-}$field has both inward and outward-propagating components (also see section 5 in Perez \& Chandran 2013). In this paper we will refer to the $\mathbf{z}_{-}$waves as the "minority" wave type, as their amplitudes are generally much smaller than those of the dominant $\mathbf{z}_{+}$waves. The nonlinear interactions between dominant and minority waves create turbulence and cause heating of the coronal plasma (Matthaeus et al. 1999).

Phenomenological models for Alfvén-wave turbulence have been developed in which the plasma heating rate $Q_{A}$ is determined by the root-mean-square (rms) values of the Elsasser variables, $Z_{ \pm} \equiv \sqrt{\left\langle\left|\mathbf{z}_{ \pm}\right|^{2}\right\rangle}$, where $\langle\cdots\rangle$ represents a spatial average (e.g., Zhou \& Matthaeus 1990; Hossain et al. 1995; Matthaeus et al. 1999; Dmitruk et al. 2001, 2002). Cranmer \& van Ballegooijen (2005) and Cranmer et al. (2007) used such a phenomenological model to develop a comprehensive description of the Alfvén waves in the solar atmosphere and fast wind, and compared the wind model with observations (also see Verdini \& Velli 2007; Verdini et al. 2010). Chandran et al. (2011) further improved the wind model by including separate energy equations for the electrons and protons, and the proton temperature anisotropy. A more advanced approach to turbulence modeling is the so-called shell model (Buchlin \& Velli 2007; Verdini et al. 2009, 2012) in which the wave spectrum is described in more detail but the nonlinear terms are still approximated.

An even more complete description of turbulence is provided by direct numerical simulations using the reduced magnetohydrodynamic (RMHD) equations. Oughton et al. (2001) and Dmitruk \& Matthaeus (2003) performed RMHD simulations for open magnetic fields, and showed that reflection-driven turbulence can be maintained in such structures. These authors neglected the effects of the solar wind outflow on the waves. Similarly, van Ballegooijen et al. (2011, hereafter paper I) simulated Alfvén wave turbulence in coronal loops, but neglected the effects of flows along the loops (also see Asgari-Targhi \& van Ballegooijen 2012; Asgari-Targhi et al. 2013, hereafter papers II and III). Perez \& Chandran (2013) were the first to perform RMHD simulations for the fast solar wind, including the effects of outflow. They solved the RMHD equations in a simulation domain that consists of a narrow magnetic flux tube with a square cross section extending from the coronal base $\left(r=1 R_{\odot}\right)$ out to the Alfvén critical point, which in their model is located at $r_{\mathrm{A}}=11.1 R_{\odot}$. Perez \& Chandran (2013) considered two values for the domain size $L_{\perp \odot}$ at the coronal base $(10 \mathrm{Mm}$ and $20 \mathrm{Mm}$ ), and different values for the correlation time of the injected waves (between 2 and $22 \mathrm{~min}$ ). The waves are 
launched with dimensionless perpendicular wavenumbers in the range $1 \leq \tilde{k}_{\perp} \leq 3$, which corresponds to actual wavenumbers $k_{\perp}=2 \pi \tilde{k}_{\perp} / L_{\perp \odot}$. Up to one third of the wave energy launched at the base is dissipated in the corona below the Alfvén critical point, and another third goes into doing work on the solar wind outflow. The remainder escapes into the heliosphere beyond the Alfvén point.

In their RMHD simulations, Perez \& Chandran (2013) found peak heating rates ranging from $10^{10} \mathrm{erg} \mathrm{g}^{-1} \mathrm{~s}^{-1}$ to $3 \times 10^{10} \mathrm{erg} \mathrm{g}^{-1} \mathrm{~s}^{-1}$. This is somewhat lower than the values found in

earlier wave-driven wind models (Cranmer et al. 2007; Verdini et al. 2010; Chandran et al. 2011), where the peak heating rates are about $3 \times 10^{11} \mathrm{erg} \mathrm{g}^{-1} \mathrm{~s}^{-1}$. The latter represents the heating rate needed to raise the coronal temperature to the observed value of about $1 \mathrm{MK}$, despite the strong cooling associated with thermal conduction and the solar wind expansion. Given that RMHD simulations provide a more accurate description of the turbulence, this suggests that the earlier models may have overestimated the ability of Alfvén wave turbulence to provide the required heating.

The purpose of the present paper is to further test the hypothesis that reflection-driven wave turbulence can provide the energy needed for heating the coronal plasma in the acceleration region of the fast solar wind. Following Perez \& Chandran (2013), we use RMHD simulations to describe the wave turbulence. The basic model is described in Section 2, but much of the details (including all of the equations) are presented in four Appendices. Simulation results are presented in Section 3, where the simulated wave dissipation rates are compared with those needed to sustain the background atmosphere. In Section 4 we consider the effects of density fluctuations, which may significantly increase the turbulent heating rate (Raymond et al. 2014). In Section 5 we present a phenomenological model for the turbulent dissipation rate. In Section 6 we discuss the implications of our results for understanding the heating and acceleration of the fast solar wind.

\section{Model for Wave-Driven Wind in an Open Flux Tube}

In this paper we consider a thin magnetic flux tube extending along the solar rotation axis at the center of the North polar coronal hole around the time of cycle minimum. We first describe the global magnetic field in which the flux tube is embedded. For this purpose we use a spherical coordinate system $(r, \theta, \phi)$, where $r$ is the radial distance from Sun center and $\theta$ is the polar angle. The magnetic field $\mathbf{B}$ is assumed to be axisymmetric with magnetic vectors that lie in meridional planes $\left(B_{\phi}=0\right)$. We assume that a current sheet is located in the equatorial plane, consistent with observations of narrow coronal streamers (e.g., Wang et al. 1997). For simplicity we assume that the current sheet extends down to the coronal base, 
i.e., we ignore the fact that coronal streamers have closed magnetic fields at lower height (e.g., Riley et al. 2011). This approximation is adequate for our present purpose because the closed fields in equatorial streamers at cycle minimum have only a minor effect on the magnetic fields over the solar poles. Above and below the equatorial plane the magnetic field is assumed to be potential, $\mathbf{B}=-\nabla \Phi$ with $\nabla^{2} \Phi=0$. For $r \gg R_{\odot}$ the field becomes nearly radial and falls off like a monopole, $B_{r} \propto r^{-2}$. The magnetic flux on the photosphere is assumed to be highly concentrated in the polar regions. In the northern hemisphere $B_{r}\left(R_{\odot}, \theta\right)=B_{\text {pole }} \cos ^{8} \theta$, where $B_{\text {pole }}$ is the net flux density at the pole (we use $B_{\text {pole }}=10$ $\mathrm{G})$. This flux distribution is consistent with observations of the Sun's magnetic field near cycle minimum (DeVore et al. 1984; Sheeley et al. 1989). Expanding $\cos ^{8} \theta$ in terms of Legendre polynomials $P_{2 n-2}(\cos \theta)$, we can extrapolate the field to larger heights. For the field line along the rotation axis $(\theta=0)$ we find

$$
B_{0}(r)=\sum_{n=1}^{5} B_{n}\left(\frac{r}{R_{\odot}}\right)^{-2 n},
$$

where $B_{n}=B_{\text {pole }}[715,2600,2160,832,128] / 6435$ with $n=1, \cdots, 5$. The field strength $B_{0}(r)$ inside the flux tube is assumed to be equal to that of the background field.

The modeled flux tube extends along the solar rotation axis from the coronal base outward into the heliosphere. The base is assumed to be located at radial distance $r_{\text {base }}=$ $1.003 R_{\odot}$, and we follow the tube out to $r_{\max }=20 R_{\odot}$. The tube is assumed to have a circular cross-section with radius $R(r)$, and magnetic flux is conserved, so $R^{2} B_{0}$ is constant along the tube. The radius of the flux tube at the coronal base is assumed to be $R_{\text {base }}=1$ $\mathrm{Mm}$, which implies that the tube is everywhere thin compared to the solar radius. The flux tube is treated as having a rigid outer boundary, and plasma flows do not penetrate this boundary. Unlike in our previous work on coronal loops (papers I, II and III), the Alfvén waves are launched by imposing transverse motions on the plasma at the coronal base, so the lower atmosphere is not included in the present model. The imposed "footpoint" motions are assumed to be incompressible and confined to the circular cross-section of the tube. Also, the footpoint motions are assumed to have a velocity amplitude $v_{\mathrm{rms}} \approx 40 \mathrm{~km} \mathrm{~s}^{-1}$, consistent with observed spectral line widths and non-thermal velocities in coronal holes (Wilhelm et al. 1998; McIntosh et al. 2008; Baneriee et al. 2009; Landi \&Cranmer 2009; Singh et al. 2011; Hahn et al. 2012; Bemporad \& Abbo 2012). The waves launched by these footpoint motions travel upward within the modeled flux tube, and dissipate their energy over a wide range of heights, heating the coronal plasma to temperatures $T_{0}(r) \sim 1 \mathrm{MK}$. The resulting gas pressure gradients are an important factor in driving the solar wind (Parker 1958, 1960). The wind has a mean outflow velocity $u_{0}(r)$ and mass density $\rho_{0}(r)$. The Alfvén waves also exert a direct force on the plasma through the wave pressure gradient, which plays a key 
role in producing the high speed of the wind emanating from corona holes Belcher 1971; Alazraki \& Couturier 1971; Hollweg 1973; Jacques 1977, 1978; Marsch \& Tu 1997). Similar effects occur in other stars (e.g., Hartmann \& MacGregor 1980). The goal of the present paper is to construct a model for the interactions between the Alfvén waves and the plasma in the fast solar wind.

The reasons for choosing $R_{\text {base }}=1 \mathrm{Mm}$ are as follows. Although the lower atmosphere is not explicitly included in our modeling, we must take into account that the magnetic field in the lower atmosphere is highly fragmented and consists of discrete flux elements surrounded by more nearly field-free plasma. In the photosphere these "flux tubes" have kilogauss field strengths and widths of order $100 \mathrm{~km}$, and they are located in the intergranulation lanes of the solar granulation pattern (e.g., Stenflo 1973; Title et al. 1987; de Wijn et al. 2009). In the photospheric regions below a polar coronal hole the average magnetic flux density is about $10 \mathrm{G}$, so the kilogauss flux tubes cover only $1 \%$ of the available area. The flux tubes expand with height in the solar atmosphere, and neighboring flux tube "merge" to form a more continuous field in the low corona (e.g., Cranmer \& van Ballegooijen 2005). A typical flux tube increases in width from about $100 \mathrm{~km}$ in the photosphere to about $1000 \mathrm{~km}$ at the coronal base (at height $\sim 2000 \mathrm{~km}$ ), hence our choice for the flux tube radius $R_{\text {base. Also, }}$ the magnetic field strength drops from about $1000 \mathrm{G}$ in the photosphere to about $10 \mathrm{G}$ in the low corona, which equals our value for the field strength $B_{\text {pole }}$ at the coronal base.

In the photosphere the magnetic flux tubes are continually shuffled about and deformed by convective flows associated with the solar granulation (Muller et al. 1994; Berger 1996; Berger et al. 1998; Chitta et al. 2012). These motions cause magnetic disturbances inside the flux tubes that propagate upward in the form of Alfvén waves and/or kink waves (e.g., Spruit 1982; Edwin \& Roberts 1983; Morton et al. 2013). Due to the density stratification of the lower atmosphere, the waves are significantly amplified on their way to the corona. The wave amplitudes increase from about $1 \mathrm{~km} \mathrm{~s}^{-1}$ in the photosphere to about $40 \mathrm{~km} \mathrm{~s}^{-1}$ in the low corona, which is crucial for producing the wave amplitudes needed to accelerate the solar wind (Cranmer et al. 2007). Therefore, we believe that the solar granulation is the main driver of the "footpoint" motions of the field lines in the low corona. These motions are not expected to be coherent from one photospheric flux tube to another. Therefore, the velocity auto-correlation length $\lambda_{\perp \odot}$ of the "footpoint" motions must be less than the flux tube radius, $\lambda_{\perp \odot}<R_{\text {base }} \sim 1 \mathrm{Mm}$. In this paper we estimate $\lambda_{\perp \odot}$ as the inverse of the perpendicular wavenumber of the imposed footpoint motions, $\lambda_{\perp \odot}=R_{\text {base }} / \tilde{k}_{\perp}$, where $\tilde{k}_{\perp}$ is the dimensionless perpendicular wavenumber. For the flux tube models considered in this paper $\tilde{k}_{\perp}=3.832$, the first zero of the $J_{1}(x)$ Bessel function, which yields $\lambda_{\perp \odot} \approx 261$ $\mathrm{km}$. While the Sun has supergranular flows on scales of 10 to $30 \mathrm{Mm}$, we believe that the correlation times of such flows (hours) are too long for the associated waves to be amplified 
in the lower atmosphere. Therefore, we do not believe that the supergranular flows can play a significant role in driving the Alfvén waves that heat and accelerate the fast solar wind.

We also require that the footpoint motions resemble a random walk, not a persistent rotational motion. The dynamical time can be defined as the time it takes for a footpoint to travel a distance equal to the flux tube diameter, $\tau_{\text {dyn }}=2 R_{\text {base }} / v_{\text {rms }}=50 \mathrm{~s}$. In the present model the footpoint motions are confined to the circular cross-section of the tube. Therefore, if the correlation time $\tau_{\mathrm{c}}$ of the velocity is much larger than $\tau_{\mathrm{dyn}}$, the footpoints move in a rotational pattern that persists for many turns before the pattern changes. We believe such persistent rotational motions are not realistic, given the randomness of the granule-scale convective flows that are ultimately responsible for the "footpoint" motions at the coronal base. Therefore, in this paper we assume $\tau_{\mathrm{c}} \approx 50 \mathrm{~s}$, comparable to the dynamical time at the coronal base. This is significantly shorter than the correlation times of 2 to 22 minutes assumed by Perez \& Chandran (2013). It is well known that short correlation times make the reflection less efficient (e.g., Dmitruk \& Matthaeus 2003), but in our view the value of $\tau_{\mathrm{c}}$ should be chosen on the basis of a model for the structure and dynamics of magnetic elements in the lower atmosphere, not the efficiency of wave reflection in the corona.

The main dissipation mechanism for the Alfvén waves is assumed to be wave turbulence. As the $\mathbf{z}_{+}$waves propagate upward along the flux tube they encounter spatial variations in Alfvén speed $v_{A}(r)$, which causes the generation of $\mathbf{z}_{-}$waves. This coupling happens not only in the chromosphere and transition region (where $v_{A}$ increases by two orders of magnitude), but also in the corona where smaller changes in Alfvén speed occur. In general the $\mathbf{z}_{-}$waves have both inward and outward-propagating components (Velli et al. 1989; Perez \& Chandran 2013). The $\mathbf{z}_{-}$waves interact with the $\mathbf{z}_{+}$waves via a well-known nonlinear process (Shebalin et al. 1983; Goldreich \& Sridhar 1995, 1997; Bhattacharjee \& Ng 2001; Maron \& Goldreich 2001; Cho et al. 2002). These nonlinear interactions create turbulence and cause a rapid transfer of wave energy to smaller and smaller spatial scales in the direction transverse to the background field. Eventually the waves reach such small scales that wave-particle interactions become important, and the wave energy is converted into heat of the background plasma. At present the details of these (collisionless) dissipation processes are not well understood, but this is not necessary for estimating the dissipation rate because in a turbulent plasma the energy cascade rate is mostly determined by the dynamics of the plasma on large spatial scales. Therefore, for simulating the overall dynamics of the solar wind it is necessary to include the effects of wave turbulence, but it is sufficient to describe the waves with relatively low spatial resolution (i.e., the turbulent waves are only partially resolved). The plasma is treated as a single fluid, i.e., differences in temperature or velocity between the various ion species are neglected, and the electron temperature is assumed to be equal to the ion temperature. 
The Alfvén waves are described in terms of their effect on the magnetic field $\mathbf{B}(\mathbf{r}, t)$ and plasma velocity $\mathbf{v}(\mathbf{r}, t)$, which are functions of position $\mathbf{r}$ within the flux tube and time $t$. The plasma motions are governed by the MHD equations:

$$
\begin{aligned}
& \frac{\partial \rho}{\partial t}+\nabla \cdot(\rho \mathbf{v})=0, \\
& \rho \frac{d \mathbf{v}}{d t}=-\nabla p+\frac{1}{4 \pi}(\nabla \times \mathbf{B}) \times \mathbf{B}-\rho \frac{G M_{\odot}}{r^{2}} \hat{\mathbf{r}}+\mathbf{D}_{v}, \\
& \rho\left[\frac{d}{d t}\left(\frac{1}{\gamma-1} \frac{p}{\rho}\right)+p \frac{d}{d t}\left(\frac{1}{\rho}\right)\right]=Q_{\mathrm{A}}-Q_{\mathrm{rad}}-Q_{\mathrm{cond}}, \\
& \frac{\partial \mathbf{B}}{\partial t}=\nabla \times(\mathbf{v} \times \mathbf{B})+\mathbf{D}_{m},
\end{aligned}
$$

where $d / d t$ is the co-moving time derivative, $\rho(\mathbf{r}, t)$ is the mass density, $p(\mathbf{r}, t)$ is the plasma pressure, $G$ the gravitational constant, $M_{\odot}$ the solar mass, $\hat{\mathbf{r}}$ is the unit vector in the radial direction, and $\mathbf{D}_{v}$ and $\mathbf{D}_{m}$ are dissipative terms. In the heat equation (44), $\gamma$ is the ratio of specific heat coefficients, $Q_{\mathrm{A}}(\mathbf{r}, t)$ is the plasma heating rate per unit volume, $Q_{\mathrm{rad}}(\mathbf{r}, t)$ is the radiative loss rate, and $Q_{\text {cond }}(\mathbf{r}, t)$ is the conductive loss rate. The latter is given by the divergence of the conductive flux, $Q_{\text {cond }}=\nabla \cdot \mathbf{F}_{\text {cond }}$, which may be positive or negative depending on position in the corona. The conductivity tensor is highly anisotropic, so the conductive flux $\mathbf{F}_{\text {cond }}$ is nearly parallel to the magnetic field $\mathbf{B}$. Since we only consider the coronal part of the flux tube, we neglect the effects of partial ionization on the internal energy of the plasma, $\gamma=5 / 3$.

In this paper we use the Reduced MHD (RMHD) equations (Strauss 1976, 1997) to simulate the dynamics of Alfvén waves in the acceleration region of the fast solar wind. The derivation of the RMHD equations for a thin flux tube with variable field strength $B_{0}(r)$ and density $\rho_{0}(r)$ was discussed in paper I. In Appendix A we discuss the modifications of the equations needed to include the effects of the solar wind outflow $u_{0}(r)$ on the waves (also see Perez \& Chandran 2013). In Appendix B we describe the numerical methods for solving these equations. We use a spectral method to describe the spatial variations of the waves in the direction perpendicular to the mean magnetic field, and finite-differences in the radial direction. The radial grid is chosen such that for outward propagating waves the wave travel time between neighboring grid points is constant with height and equal to the time step of the simulation, $\Delta t=1 \mathrm{~s}$. This allows the waves to propagate to large height without numerically induced distortions. The key feature of the RMHD equations is that they retain the nonlinear terms responsible for the development of Alfvén wave turbulence. To dissipate the waves, artificial damping is used at high parallel and perpendicular wavenumbers. For simplicity the same damping rates are used for both the magnetic- and velocity fluctuations [see equations (B1) and (B2)], so the magnetic Prandtl number (ratio of viscosity to magnetic 
diffusivity) satisfies $\operatorname{Pr}_{\mathrm{M}}=1$. In this paper we assume that all heating occurs via Alfvén wave turbulence, but it remains to be seen whether such heating is indeed sufficient to explain the observed properties of the fast solar wind.

Unlike in our modeling of coronal loops in active regions (papers I, II and III), we do not include the lower solar atmosphere in the present version of the RMHD model. The main reason is that we need to simulate the waves for longer periods than in our earlier loop simulations, and including the lower atmosphere would require short time steps $(\Delta t \sim 0.1 \mathrm{~s})$, which is not practical for simulating the solar wind with the present version of our RMHD code. Therefore, the lower boundary of the RMHD model is assumed to be located at the coronal base where the temperature is $T_{0} \approx 0.3 \mathrm{MK}$.

The outward- and inward propagating Alfvén waves travel with velocities $u_{0} \pm v_{A}$, respectively. Hence, the waves are significantly affected by the outflow when $u_{0}$ becomes comparable to $v_{A}$. In fact, at heights above the Alfvén critical point (where $u_{0}>v_{A}$ ) the "inward" propagating waves actually move radially outward with a velocity less than the plasma velocity $u_{0}$. Conversely, the Alfvén waves have significant effects on the plasma: (1) the wave turbulence causes plasma heating, and (2) the waves exert a direct force on the out-flowing plasma via wave pressure gradients. The latter is thought to play an important role in producing the high speed of the wind emanating from coronal holes (Hollweg 1973; Marsch \& Tu 1997). Therefore, in the acceleration region of the wind the plasma and waves must exchange energy with each other. The details of this energy exchange are discussed in Appendix C.

Three-dimensional RMHD modeling requires that we first set up a one-dimensional model for the background atmosphere inside the flux tube. In addition to the magnetic field strength $B_{0}(r)$ given in equation (1), we also need the plasma temperature $T_{0}(r)$, density $\rho_{0}(r)$ and outflow velocity $u_{0}(r)$. Appendix $\mathrm{D}$ describes how these quantities are computed. The temperature is a prescribed function of $r$ and is given by equation (D10). Using a formula for temperature has the advantage that higher derivatives can be accurately computed, which is important for evaluating the energy loss rates due to thermal conduction. We require that the background model satisfy not only the mass conservation equation (C8) but also the equation of motion (C9). The latter includes the wave pressure force $D_{\mathrm{wp}}(r)$, so we need an approximation of this force that can be used in constructing the background model. Appendix D describes how this approximation is obtained from the wave action equation (D3), which is an approximation for the wave energy equation. We also describe how the approximation for $D_{\mathrm{wp}}$ is used in the iterative process for deriving the outflow velocity $u_{0}(r)$.

In this paper we distinguish between the wave energy dissipation rate $Q_{\text {tot }}$ and the plasma heating rate $Q_{\mathrm{A}}$. If the waves provide all the heating, these two rates should be 
equal, $Q_{\mathrm{tot}}=Q_{\mathrm{A}}$. The goal of the present work is to construct a solar wind model for which this condition is indeed satisfied. If the condition is satisfied, we can derive the full energy equation by adding equations (C16) and (C18) from Appendix C:

$$
B_{0} \frac{d}{d r}\left(\frac{F_{\text {total }}}{B_{0}}\right)=-Q_{\text {rad }},
$$

where $F_{\text {total }}=F_{\text {plasma }}+F_{\text {waves }}$ is the total energy flux along the tube. It follows that in the absence of radiative losses the total energy flow $\pi R^{2} F_{\text {total }}$ is constant along the tube. This demonstrates the consistency of the equations used in this paper: except for radiative losses, the total energy of the system (plasma + waves) is conserved. However, in the next Section we will consider a model with a smooth background atmosphere for which the condition $Q_{\mathrm{A}}=Q_{\mathrm{tot}}$ is not satisfied. In this case the model is not consistent from an energy point of view.

\section{Model with a Smooth Background Atmosphere}

In this section we describe results for a reflection-driven wave-turbulence model of the fast solar wind. Figure 1 shows the structure of the background atmosphere. Figure 1)(a) shows the magnetic field strength $B_{0}(r)$ as computed from equation (1) with $B_{\text {pole }}=10 \mathrm{G}$. Note that $B_{0}(r)$ drops off faster than $r^{-2}$, which is due to the $\cos ^{8} \theta$ distribution of magnetic flux on the photosphere. Figure 1(b) shows the flux tube radius $R(r)$, which is computed from flux conservation. Note that $R(r)$ increases from $1 \mathrm{Mm}$ at the coronal base to $60 \mathrm{Mm}$ at $r=20 R_{\odot}$. Figure 1(c) shows the temperature $T_{0}(r)$ computed from equation (D10) with the parameters $C_{0}=0.35, C_{1}=2, m=0.3$, and $k=8$. This formula is intended to give only a qualitative description of the temperature structure in coronal holes, and does not represent an atmosphere in thermal balance. Note that $T_{0}(r)$ increases from about $0.3 \mathrm{MK}$ at the coronal base, and reaches a maximum of about $1.31 \mathrm{MK}$ at $r \approx 3 R_{\odot}$. At large heights the temperature decreases as $T_{0} \propto r^{-0.3}$.

All other quantities shown in Figure 1 are computed as described in Appendix D. We include the effects of the wave pressure force $D_{\mathrm{wp}}$ on the background plasma. Figure 1(d) shows the wave energy density $U_{\mathrm{A}}(r)$, which is determined by solving the wave action equation (D3). The assumed amplitude of the waves at the coronal base is $v_{\text {rms }}\left(r_{\text {base }}\right)=40.6$ $\mathrm{km} \mathrm{s}^{-1}$. Note that $d U_{\mathrm{A}} / d r<0$, which implies that the waves produce an outward force on the plasma $\left(D_{\mathrm{wp}}>0\right)$. Figure 1(e) shows the wave action parameter $S_{\mathrm{A}}(r)$ defined in equation (D4). The wave action decreases by about $50 \%$ over the height range of the model, which is due to wave dissipation. For the purpose of constructing the background model the wave dissipation rate is assumed to be equal to the plasma heating rate $Q_{\mathrm{A}}$, and is computed 
by evaluating the energy losses of the plasma [see equations (C11) through (C14)]. Figure $1(f)$ shows $Q_{\mathrm{A}}(r)$ together with its contributions from radiative losses (blue curve), thermal conduction (red curve), and the advection terms in the heat equation (green curve). Note that radiative losses are important only in the low corona, and advection dominates in the region $1.1-2.7 R_{\odot}$. At $r>10 R_{\odot}$ the conductive losses are negative (dashed red curve), indicating the plasma is heated by conduction from below. Figure $1(\mathrm{~g})$ shows the acceleration $D_{\mathrm{wp}} / \rho_{0}$ due to the wave pressure force (red curve) together with the acceleration of gravity (black curve). Note that the wave pressure acceleration exceeds the gravitational acceleration already at $r=2 R_{\odot}$.

Figure1(h) shows the outflow velocity $u_{0}(r)$ (black curve) as computed with the iterative method described in Appendix D, and Figure 1(i) shows the corresponding density $\rho_{0}(r)$ from mass flux conservation. Here we assumed a coronal base pressure $p_{0 \text {,base }}=0.1$ dyne $\mathrm{cm}^{-2}$, which yields a base density $\rho_{0, \text { base }}=2.32 \times 10^{-15} \mathrm{~g} \mathrm{~cm}^{-3}$. The Alfvén speed $v_{A}(r)$ is plotted as the red curve in Figure $1(\mathrm{~h})$, and reaches a maximum of about $2000 \mathrm{~km} \mathrm{~s}^{-1}$ at $r=1.5$ $R_{\odot}$. The red and black curves cross at $r=7.13 R_{\odot}$, which is the Alfvén critical point. At the outer boundary of the model $\left(r=20 R_{\odot}\right)$ the outflow velocity reaches about 800 $\mathrm{km} \mathrm{s}^{-1}$, typical for the terminal velocity of the fast solar wind. The critical point of the flow is located at $r_{\mathrm{c}}=1.83 R_{\odot}$, which is significantly smaller than the value $r_{\mathrm{c}}=4.48 R_{\odot}$ that would exist in the absence of wave pressure forces (i.e., with only $d p_{0} / d r$ driving the flow). Therefore, the wave pressure force $D_{\mathrm{wp}}$ plays a major role in producing the fast solar wind (e.g. Belcher 1971; Hollweg 1973; Jacques 1978).

\subsection{RMHD Simulations}

The atmospheric model presented in Figure 1 is used as the "background" for threedimensional, time-dependent RMHD simulations of the Alfvén waves inside the flux tube. The numerical methods for solving the RMHD equations are described in Appendix B. In the initial state there are no waves at any height. The waves are launched by imposing certain "footpoint" motions on the plasma at the coronal base, which is the lower boundary of the RMHD model. The imposed velocity patterns are a combination of two basis functions $F_{k}(\xi, \varphi)$ as described in Appendix B (also see Appendix B of paper I). In our model these "driver modes" have indices $k=10$ and $k=11$, and both modes have dimensionless wavenumber $a_{k}=3.832$, given by the first zero of the $J_{1}(x)$ Bessel function. Also, both modes have azimuthal mode number $m_{k}=1$, but with different directions of the flow in the perpendicular plane. The amplitudes $f_{k}\left(r_{\text {base }}, t\right)$ of the driver modes vary randomly with time $t$ in the simulation. For each mode we first create a normally distributed random se- 
quence $f(t)$ on a grid of times covering the entire simulation $\left(t_{\max }=30,000 \mathrm{~s}\right)$. Then the sequence is Fourier filtered using a Gaussian function $G(\tilde{\nu})=\exp \left[-\left(\tau_{0} \tilde{\nu}\right)^{2}\right]$, where $\tilde{\nu}$ is the temporal frequency (in $\mathrm{Hz}$ ) and $\tau_{0}$ is a specified parameter. In the present work we take $\tau_{0}=120 \mathrm{~s}$, which corresponds to a correlation time $\tau_{\mathrm{c}}=\tau_{0} / \sqrt{2 \pi} \approx 48 \mathrm{~s}$. The filtered sequence is renormalized such that the rms vorticity of each mode is $\omega_{\text {rms }}=0.11 \mathrm{rad} \mathrm{s}^{-1}$; this corresponds to a combined velocity amplitude $v_{\mathrm{rms}}=40.6 \mathrm{~km} \mathrm{~s}^{-1}$, consistent with the value used in the setup of the background model. Note that the correlation time $\tau_{\mathrm{c}}$ is comparable to the dynamical time of the footpoint motions, $\tau_{\text {dyn }}=2 R_{\text {base }} / v_{\text {rms }}=49.3 \mathrm{~s}$. Therefore, the footpoints are strongly intermixed on the correlation timescale, leading to strong turbulence in the Alfvénic fluctuations.

The simulated waves are described in terms of mode amplitudes $h_{k}(r, t)$ and $f_{k}(r, t)$, which represent the magnetic- and velocity fluctuations, respectively (see Appendix B). The waves are also described with Elsasser-like variables, $\omega_{ \pm, k}(r, t)$, which represent the vorticities in the $\mathbf{z}_{ \pm}$waves. The dominant $\omega_{+, k}$ waves always travel outward, but the minority $\omega_{-, k}$ waves can have both inward and outward-propagating components (Velli et al. 1989). The linear coupling between the $\omega_{+, k}$ and $\omega_{-, k}$ waves is described by the second and third terms in equation (B33). This coupling is due to spatial variations in Alfvén speed $v_{A}(r)$, density $\rho_{0}(r)$, field strength $B_{0}(r)$, and outflow velocity $u_{0}(r)$. The outward-propagating waves first reach the outer boundary of the RMHD model $\left(r=20 R_{\odot}\right)$ after about 10,891 s. The waves are simulated for a period of 30,000 s to ensure that a statistically stationary state is reached. In this state there are dominant $\omega_{+, k}$ waves and minority $\omega_{-, k}$ waves at all heights, and the waves have a broad spectrum of perpendicular wavenumbers, indicating that strong wave turbulence has developed. We find that the minority waves mainly travel outward with the same velocity $\left(u_{0}+v_{A}\right)$ as the dominant waves, consistent with the predictions of Velli et al. (1989). Therefore, it is not correct to think of the $\omega_{-, k}$ waves as inward-propagating waves.

Figure 2 shows various wave-related quantities averaged over the cross-section of the flux tube and over the time. Each quantity is averaged over the time interval $t_{0}(r)+300 \leq$ $t \leq 30000$ (in seconds), where $t_{0}(r)$ is the time for an outward propagating wave to reach a certain height. The black curve in Figure 2(a) shows the rms velocity amplitude of the waves, $v_{\text {rms }}(r)$, which reaches a peak value of about $330 \mathrm{~km} \mathrm{~s}^{-1}$ at $r \approx 9 R_{\odot}$. Comparing with Figure 1(h), we see that $v_{\text {rms }}<v_{A}$ at most heights, but $v_{\text {rms }} \sim v_{A}$ near the outer boundary of the model at $r=20 R_{\odot}$. This indicates that the RMHD approximation begins to break down at that height. The solid red and green curves in Figure 2(a) show the rms values of the Elsasser variables, $Z_{ \pm}(r)=\sqrt{\left\langle\left|\mathbf{z}_{ \pm}\right|^{2}>\right.}$, where $\mathbf{z}_{ \pm} \equiv \mathbf{v}_{1} \mp \mathbf{B}_{1} / \sqrt{4 \pi \rho_{0}}$. Note that the minority $Z_{-}$waves are are much weaker than the dominant $Z_{+}$waves; at $r>5 R_{\odot}$ the ratio $Z_{-} / Z_{+}<0.01$, consistent with the results of Cranmer et al. (2007). The amplitude $Z_{-}(r)$ of the minority waves has a sharp minimum at $r \approx 1.3 R_{\odot}$. We attribute this to the fact 
that the Alfvén speed has a maximum near that height [see Figure 1(h)], which reduces the magnitude of the second term in equation (B3) and thereby the amplitude of the minority waves. The Elsasser variable of the minority waves, $Z_{-}$, is significantly smaller in our model compared to Perez \& Chandran (2013), probably because we use driver waves with shorter correlation times (i.e., shorter wavelength and less reflection).

The dashed red and green curves in Figure 2(a) give the Elsasser variables for a different "linear" model in which the nonlinear and damping terms in the RMHD equations (B1) and (B2) are omitted. In that case there is no turbulent cascade, so the level of minority waves is determined solely by the linear (reflection) terms in the equations. Comparing the solid and dashed green curves in Figure 2(a), we see that one effect of the nonlinear terms is to suppress the amplitude of the minority waves by a factor of 3 to 10 compared to the linear model. This comparison between the two models shows that the amplitude of the minority waves is determined by two processes: (1) linear coupling with the dominant waves, and (2) decay of the minority waves due to turbulence. The linear process (1) can lead to either production or destruction of minority waves, depending on whether the ratio $Z_{-} / Z_{+}$is smaller or larger than the value $\left(Z_{-} / Z_{+}\right)_{\text {lin }}$ found in a purely linear model (i.e., model without nonlinear terms). In such a linear model only the first process operates, and a certain level of minority waves is obtained, as shown by the dashed green curve in Figure 2(a). This level of $Z_{-}$is already low compared to $Z_{+}$because the reflection is relatively weak in our "smooth" model. With the nonlinear terms switched on, $Z_{-}$is further reduced as shown by the solid green curve. This additional reduction is due to the second process, turbulent decay of minority waves. The reduction is significant because the minority waves have a short nonlinear time scale (see Section 5). Therefore, the nonlinear interactions play an important role in determining the amplitudes of the minority waves (also see Chandran \& Hollweg 2010).

Figure2(b) shows the rms vorticity of the waves, i.e., the component of vorticity parallel to the background field. This quantity is dominated by waves with higher perpendicular wavenumbers, and therefore is sensitive to the spatial resolution of the model. Note that the vorticity decreases with height, which is due to the expansion of the flux tube with height (a similar effect was found for coronal loops, see paper II). Figure 2(c) shows the rms value of the magnetic fluctuations of the waves, $B_{1, \mathrm{rms}}(r)$. Comparing with Figure 1(a), we see that $B_{1, \mathrm{rms}} \approx B_{0}$ near the outer boundary of the model, again indicating that the RMHD approximation begins to break down at that height.

Figure2(d) shows the total energy density $U_{\text {tot }}$ of the simulated waves (full black curve), together with the contributions to this quantity from the kinetic energy $U_{\text {kin }}$ (red curve) and magnetic energy density $U_{\text {mag }}$ (green curve). The dashed curve shows the energy density $U_{\mathrm{A}}$ 
used in the setup of the background model [same as Figure1(d)]. We see that $U_{\text {kin }} \approx U_{\text {mag }}$ and $U_{\text {tot }} \approx U_{\mathrm{A}}$, so the assumptions made in the model setup (see Appendix D) seem consistent with the wave simulation results.

Figure 2(e) shows the total energy dissipation rate $Q_{\text {tot }}(r)$ of the simulated turbulence (solid black curve). This rate is given by $Q_{\text {tot }}=Q_{\perp}+Q_{\|}$, where $Q_{\perp}$ is the contribution from damping at high perpendicular wavenumbers (green curve), and $Q_{\|}$is the contribution from damping at high parallel wavenumbers (red curve), see equations (다) and (C7). Note that at large heights the parallel contribution is larger than the perpendicular one; this is due to the weakness of the turbulence for the dominant waves in the present model. The dashed black curve shows the plasma heating rate $Q_{\mathrm{A}}$ used in the model setup [same as the black curve in Figure 1(f)]. Figure 2(f) shows the same heating rates per unit mass, $Q_{\text {tot }} / \rho_{0}$ (full black curve) and $Q_{\mathrm{A}} / \rho_{0}$ (dashed curve). Note that $Q_{\text {tot }} / \rho_{0}$ has a minimum at $r \approx 1.3 R_{\odot}$, near the height where the Alfvén speed has its maximum and the amplitude of the minority waves is reduced. Figures 2(e) and 2(f) show that over a wide range of heights the dissipation rate $Q_{\text {tot }}(r)$ is significantly smaller than the plasma heating rate $Q_{\mathrm{A}}(r)$ needed to sustain the background atmosphere. We conclude that for the smooth model considered here the simulated wave turbulence does not provide enough heating to raise the temperature to the assumed level $T_{0}(r)$ shown in Figure 1(c).

\subsection{Power Spectra and Wave Frequencies}

Figures 3(a) and 3(b) show power spectra for the Elsasser variables as function of dimensionless perpendicular wavenumber $a_{\perp}$ for four different heights in the model. For each height we compute the wave power in individual modes with wavenumbers $a_{k}(k=1, \cdots, 209)$, and then collect the results into bins in wavenumber space with $\Delta a_{\perp}=2$ (for more details on how such spectra are computed, see section 4.2 of paper I). These results are derived from the last 800 time steps of the simulation. Figure 3(a) shows the power spectra for the outward waves. The first and highest bin for each curve $\left(a_{\perp} \approx 3\right)$ represents the outer scale of the turbulence, and contains the driver modes $\left(a_{k}=3.832\right)$ that are launched at the coronal base and propagate upward in height. The remaining bins are filled by reflection-driven turbulent cascade. The sharp drop in power at $a_{\perp}=15$ is due to the onset of damping $\nu_{k}$ at that wavenumber. At lower wavenumbers (where $\nu_{k}=0$ ) the power spectra are rather flat, dropping only about one order of magnitude over the wavenumber range $2<a_{\perp}<15$. This flatness of the spectrum may be due to a "bottleneck effect" resulting from the use of a damping rate $\nu_{k}$ that increases strongly with perpendicular wavenumber (e.g., Beresnyak \& Lazarian $2009 \mathrm{~b})$. However, when considering the full wavenumber range the power spectrum for out- 
ward waves is quite steep: the power drops by eight orders of magnitude over the range $2<a_{\perp}<30$. Figure 3(b) shows similar spectra for the minority waves, which have a much shallower spectrum. Note that at low wavenumbers the minority wave power is much smaller than the dominant wave power. For example, at $r=6 R_{\odot}$ (dashed curve) the power ratio is about $10^{-4}$, consistent with $Z_{-} / Z_{+} \sim 10^{-2}$ at that height in Figure 2(a).

We also compute temporal power spectra of dominant and minority waves, and derive the average wave frequency $\tilde{\omega}_{ \pm}$as function of dimensionless perpendicular wavenumber $a_{\perp}$. The results are shown in Figures 3 (c) and 3)(d) for four different heights in the model. At the outer scale of the turbulence $\left(a_{\perp} \approx 3\right)$ the outward waves are dominated by the driver modes. Using the model for random footpoint motions described in Section 3, we find that the driver waves have an average frequency $\tilde{\omega}_{+} \approx 2 \sqrt{\pi} \tau_{0}^{-1} \approx 0.03 \mathrm{rad} \mathrm{s}^{-1}$, where $\tau_{0}(=120$ s) is the parameter used in the setup of the random sequence. The left-most point on the curve for $r=1.2 R_{\odot}$ in Figure 3(c) is consistent with the expected value of frequency for the driver waves. Note that the wave frequencies $\tilde{\omega}_{ \pm}$generally increase with perpendicular wavenumber $a_{\perp}$ for both dominant and minority waves. Also, the frequencies of the dominant waves are somewhat larger than the frequencies of the minority waves, even though both travel outward.

We now consider the question whether the turbulence in our model is weak or strong. The turbulence is caused by nonlinear interactions between Alfvén waves (Iroshnikov 1963; Kraichnan 1965). Following Chandran et al. (2009), we consider the interactions between two wave packets $\mathbf{z}_{ \pm}$at the outer scale $\lambda_{\perp}$ of the turbulence, which we take to be the inverse of the perpendicular wavenumber of the driver waves, $\lambda_{\perp}=R / 3.832$. The rate of shearing of the $\mathbf{z}_{ \pm}$wave packet by the $\mathbf{z}_{\mp}$ wave packet is given by $\tilde{\omega}_{\text {shear, } \mp}=Z_{\mp} / \lambda_{\perp}$. The waves interact for a certain "collision" time, which we take to be the inverse of the wave frequency in the comoving frame, $t_{\mathrm{coll}, \mp}=1 / \tilde{\omega}_{0, \mp}^{\prime}$, where the subscript 0 indicates the outer scale.

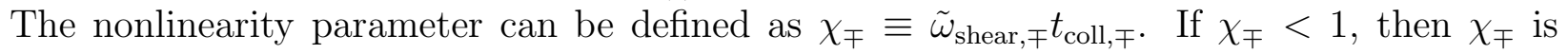
approximately the fractional change in the outer-scale $\mathbf{z}_{ \pm}$wave packet due to shearing by the outer-scale $\mathbf{z}_{\mp}$ wave packet (Chandran et al. 2009). We find that for the "smooth" model discussed in this Section, the shearing rate $\tilde{\omega}_{\text {shear,- }}$ relevant for the cascade of the dominant waves varies from about $0.04 \mathrm{rad} \mathrm{s}^{-1}$ at $r=1.1 R_{\odot}$ to less than $4 \times 10^{-4} \mathrm{rad} \mathrm{s}^{-1}$ at $r>10 R_{\odot}$. At larger heights these shearing rates are significantly smaller than the frequencies $\tilde{\omega}_{0,-}^{\prime}$ for the minority waves, which determine the "collision" time for dominant waves. In contrast, the shearing rate $\tilde{\omega}_{\text {shear,+ }}$ relevant for the cascade of the minority waves is comparable to the frequencies $\tilde{\omega}_{0,+}^{\prime}$ for the dominant waves. Therefore, in the present model the dominant outward waves have large amplitudes but undergo weak turbulence $\left(\chi_{-} \ll 1\right)$, whereas the minority waves have small amplitudes but undergo strong turbulence $\left(\chi_{+} \sim 1\right)$. This has important consequences for the wave dissipation rate (see Section 5). 
The power spectra shown in Figures [3(a) and 3(b) can be compared with results from high-resolution simulations of anisotropic MHD turbulence in a uniform background atmosphere. Here we focus on models in which there is a large imbalance between counterpropagating Alfvén waves (e.g., Beresnyak \& Lazarian 2008, 2009a, b; Perez \& Boldyrev 2009; Perez et al. 2012). In such models the energy is injected by random forcing of the waves at low perpendicular wavenumbers throughout the computational domain. For example, Perez et al. (2012) found that the spectra for the inertial range are well fit by power laws with exponents of about $-3 / 2$ for the dominant waves and slightly steeper for the subdominant waves. In contrast, in the present model the background atmosphere is highly inhomogeneous, the outward waves are launched at the coronal base, and minority waves are produced only by wave reflections (also see Perez \& Chandran 2013). We find much steeper spectra than in the homogeneous turbulence models, but this is likely due to the relatively low spatial resolution of the model presented here. This is confirmed by the work of Perez \& Chandran (2013), who used higher resolution and obtained spectra similar to those found in the homogeneous models. Therefore, the spectra shown in Figures 3 (a) and (b) are probably not realistic. However, the main focus of our study is the wave dissipation rate $Q_{\text {tot }}$, and this quantity is likely to be much less affected by limited spatial resolution.

\section{Model With Density Fluctuations}

The solar wind model discussed in Section 3 has the problem that the Alfvén wave dissipation rate $Q_{\text {tot }}(r)$ predicted by the RMHD simulation is much smaller than the heating rate $Q_{\mathrm{A}}(r)$ needed to sustain the background atmosphere. Therefore, the model is not consistent from an energy point of view. To obtain a more consistent model we must find a way to increase $Q_{\text {tot }}$ by about a factor $\sim 5$ without also increasing $Q_{\mathrm{A}}$. In a reflectiondriven wave-turbulence model the wave dissipation rate may be increased by creating more wave reflection. We suggest that in the acceleration region of the solar wind there are MHD waves of various type, not only Alfvén waves but also compressive, slow-mode waves traveling with velocities of the order of the sound speed, $c_{s} \sim 100 \mathrm{~km} \mathrm{~s}^{-1}$. These sound waves may in fact be produced by coupling with the Alfvén waves (e.g., Kudoh \& Shibata 1999; Morivasu et al. 2004; Matsumoto \& Shibata 2010), and such coupling may also be involved in the formation of jets and spicules (Tian et al. 2014; Cranmer \& Woolsey 2015). Sound waves have associated density fluctuations, $\delta \rho(r, t)$, and since the magnetic field $B_{0}(r)$ is relatively unaffected, the Alfvén speed $v_{A}(r, t)$ will also vary in space and time. The Alfvén waves reflect due to gradients in Alfvén speed, $d v_{A} / d r$ [see equation (B3)], and in the presence of density fluctuations these gradients may be significantly enhanced. Therefore, sound waves with sufficient amplitude may act to "scatter" the dominant, outward propagating Alfvén 
waves, producing more minority waves and thereby enhancing the turbulent dissipation rate.

Observational evidence for density fluctuations comes from a variety of sources. Radio observations have long been used to detect density fluctuations in the solar wind (e.g., Coles \& Harmon 1989; Woo 1996). Spangler (2002) used radio interferometry data to detect density variations with an amplitude of $6 \%-15 \%$ at heliocentric distances of $16-26 R_{\odot}$. White light eclipse images show a variety of coronal density structures (e.g., Druckmüller et al. 2014). Raymond et al. (2014) used the Atmospheric Imaging Assembly (AIA) on the Solar Dynamics Observatory (SDO) to observe striations in the tail of the sun-grazing Comet Lovejoy. These striations indicate the presence of large density variations (at least a factor six) between neighboring coronal flux tubes on scales of a few thousand kilometers. Most of these observations refer to density variations across magnetic field lines. However, Krishna Prasad et al. (2012) detected long-period intensity oscillations in open coronal structures observed with AIA, and interpreted the results in terms of slow-mode waves propagating along field lines. Mivamoto et al. (2014) used spacecraft radio occultation measurements at heights $1.5<r<20.5 R_{\odot}$, and found quasi-periodic density disturbances with periods of $100-2000 \mathrm{~s}$ and amplitudes of $30 \%$ at $r=5 R_{\odot}$, which may also be due to slow-mode waves. Using data from AIA, Tian et al. (2011) found signatures of both longitudinal and transverse waves in plume-like structures, rooted in magnetized regions of the quiet solar atmosphere. The longitudinal waves have typical periods of 5 - 15 minutes and a phase speed of 120 $\mathrm{km} \mathrm{s}^{-1}$. Threlfall et al. (2013) compared wave observations from the Coronal Multi-channel Polarimeter (CoMP) and AIA/SDO, and found evidence for transverse waves with periods of 3 - 8 minutes and longitudinal waves with period of 6 - 11 minutes. In a similar study, Liu et al. (2015) found longitudinal waves with periods of 10 - 20 minutes and a phase speed of $120 \mathrm{~km} \mathrm{~s}^{-1}$. The associated intensity oscillations have amplitudes of only $1 \%$, but this is likely due to line-of-sight integration effects. A period of 15 minutes corresponds to a parallel wavelength of about $0.16 R_{\odot}$. Based on such measurements we assume density variations along the magnetic field with an rms amplitude of $10 \%$ and an auto-correlation length of $0.04 R_{\odot}$, one quarter of the typical wavelengths observed by Tian et al. (2011) and Liu et al. $(2015)$.

In this section we consider a simple model for the effect of the density fluctuations on the Alfvén waves. For simplicity the density variations are assumed to be static, i.e., independent of time. We construct a model with spatial variations in density $\delta \rho(r)$ by taking the solution from the "smooth" model described in Section 3 and adding variations to certain physical quantities. The resulting outflow velocity $u_{0}^{\prime}(r)$, Alfvén speed $v_{A}^{\prime}(r)$, and density $\rho_{0}^{\prime}(r)$ are shown in Figures 4(a) and 4(b). The density is given by $\rho_{0}^{\prime}(r)=\rho_{0}(r)[1+\epsilon(r)]$, where $\rho_{0}(r)$ is the density in the smooth model, and $\epsilon(r) \equiv \delta \rho / \rho_{0}$ is a random function of position (see below). The temperature $T_{0}(r)$ and magnetic field strength $B_{0}(r)$ are assumed to be 
unaffected by the fluctuations, but the Alfvén speed $v_{A}^{\prime}(r)=v_{A}(r)[1+\epsilon(r)]^{-1 / 2}$, where $v_{A}(r)$ is the Alfvén speed in the smooth model. To conserve mass and maintain the same mass flux as in the smooth model, we assume that the outflow velocity $u_{0}^{\prime}(r)=u_{0}(r) /[1+\epsilon(r)]$. We make slight adjustments to $\epsilon(r)$ near the Alfvén critical point to ensure that this point is crossed only once, i.e., there is only a single point $r_{A}$ in the model where $u_{0}^{\prime}\left(r_{A}\right)=v_{A}^{\prime}\left(r_{A}\right)$. We found this adjustment is necessary to avoid pile-up of "inward" waves near the Alfvén critical point.

The random function $\epsilon(r)$ is constructed as follows. We first create a normally distributed random sequence $\epsilon^{\prime}(r)$ on a grid that is uniform in radial distance. Then the sequence is Fourier filtered using a Gaussian function $G\left(k_{r}\right)=\exp \left[-\left(\lambda_{0} k_{r} / 2 \pi\right)^{2}\right]$, where $k_{r}$ is the radial wavenumber, and $\lambda_{0}$ is a parameter that determines the correlation length of the density variations. In this paper we take $\lambda_{0}=0.1 R_{\odot}$, which corresponds to a correlation length $\lambda_{\mathrm{c}}=\lambda_{0} / \sqrt{2 \pi} \approx 0.04 R_{\odot}$. The filtered sequence is then renormalized such that $\epsilon_{\mathrm{rms}}=0.1$ and remapped onto the radial grid $r_{n}$ used for the numerical simulation. In this model both the correlation length $\lambda_{\mathrm{c}}$ and fluctuation amplitude $\epsilon_{\mathrm{rms}}$ are assumed to be independent of height.

The resulting one-dimensional model with spatial density variations is used as the background atmosphere for three-dimensional RMHD simulations of the Alfvén waves. The boundary conditions and method of solution of the RMHD equations are exactly the same as for the smooth model discussed in Section 3. We find that in the model with density variations the minority waves have both inward and outward-propagating components. Figure 4(c) shows the simulation results for the rms velocity amplitude of the waves (black curve) and the Elsasser variables for dominant waves (red curve) and minority waves (green curve). These results have been averaged over the cross-section of the flux tube and over time. Note that the time-averaged Elsasser variable $Z_{-}(r)$ for the minority waves shows strong spatial variations, especially near the Alfvén critical point where the "inward" waves are nearly stationary. The mean value of $Z_{-}(r)$ is significantly increased compared to that in the smooth model [compare with Figure 2 (a)], and the dip in $Z_{-}(r)$ near $r=1.3 R_{\odot}$ is no longer present. This enhancement of the minority waves is due to additional scattering that occurs in the model with density fluctuations. The Elsasser variable $Z_{+}(r)$ for the dominant waves [red curve in 4(c)] shows much smaller spatial variations, and the mean value is nearly unchanged from that in the smooth model.

Figure 4(d) shows the total energy density $U_{\text {tot }}(r)$ of the simulated waves (solid black curve), together with the contributions from kinetic energy (red curve) and magnetic energy (green curve). Note that the fluctuations in these quantities are relatively small, which is due to the fact that they are dominated by the outward-propagating waves. The dashed curve 
in Figure 4(d) again shows the energy density $U_{\mathrm{A}}(r)$ used in the setup of the background model [same as in Figure 1(d)].

Figure $4(\mathrm{e})$ shows the energy dissipation rate $Q_{\text {tot }}(r)$ as derived from the RMHD simulations (solid black curve), together with the contributions from $Q_{\perp}$ (green curve) and $Q_{\|}$ (red curve). These results are averaged over the cross-section of the flux tube and over time. Note that the dissipation rates have large spatial variations that are correlated with those of the Elsasser variable $Z_{-}(r)$ shown in Figure 4(c). Comparing Figures 2(e) and 4(e), we see that the dissipation rate $Q_{\text {tot }}(r)$ is significantly increased compared to its value in the smooth model. The dashed curve in Figure 4(e) shows the plasma heating rate $Q_{\mathrm{A}}(r)$ needed to sustain the background atmosphere. We see that the mean value of $Q_{\text {tot }}(r)$ approximately equals the heating rate $Q_{\mathrm{A}}(r)$ at all heights up to $r=8 R_{\odot}$, and exceeds $Q_{\mathrm{A}}$ at larger heights, i.e., in the model with density fluctuations the simulated wave turbulence produces enough energy dissipation to heat the background atmosphere. The same can be seen in Figure 4(f), where we plot the dissipation rate per unit mass, $Q_{\text {tot }} / \rho_{0}^{\prime}$ (solid black curve), and the heating rate per unit mass, $Q_{\mathrm{A}} / \rho_{0}^{\prime}$ (dashed black curve). For heights in the range 1 to $10 R_{\odot}$ the mean dissipation rate is about $10^{11} \mathrm{erg} \mathrm{g}^{-1} \mathrm{~s}^{-1}$, similar to the values found in the models by Cranmer et al. (2007) (see their Figure 7) and Chandran et al. (2011) (their Figure $3 \mathrm{~b}$ ). However, these earlier models did not include the effects of density fluctuations on the reflection of the Alfvén waves.

\section{Phenomenology for Turbulence in an Inhomogeneous Atmosphere}

Several authors have developed "phenomenological" models for the dissipation rate $Q$ in homogeneous MHD turbulence. For decaying turbulence the dissipation rate may be approximated as

$$
Q_{\text {phen }}=\rho_{0} \frac{Z_{+}^{2} Z_{-}+Z_{-}^{2} Z_{+}}{4 \lambda_{\perp}}
$$

where $Z_{+}$are the rms values of the Elsasser variables, and $\lambda_{\perp}$ is the outer scale of the turbulence (Hossain et al. 1995). The same expression has been applied for reflection-driven turbulence in the solar wind (e.g., Zhou \& Matthaeus 1990; Matthaeus et al. 1999; Dmitruk et al. 2001, 2002). Dmitruk \& Matthaeus (2003) found good agreement between the above phenomenological model and results from numerical RMHD simulations. Cranmer et al. (2007) and Chandran et al. (2011) constructed detailed models of the fast solar wind based on such expressions for the wave dissipation rate.

Equation (77) is based on the assumption that the cascade times for dominant and minor-

ity waves are given by the nonlinear time scales, $t_{\mathrm{nl}, \pm} \equiv \lambda_{\perp} / Z_{\mp}$. Chandran et al. (2009) con- 
sider reflection-driven turbulence and argue that these expressions are appropriate when the dominant waves undergo weak turbulence $\left(\chi_{-} \ll 1\right)$ and the minority waves undergo strong turbulence $\left(\chi_{+} \sim 1\right)$, as is the case in our "smooth" model (Section 3). Perez \& Boldyrev (2009) and Mallet et al. (2015) use a different expression for the nonlinear time that also includes a dependence on the alignment angle $\theta$ between the $\mathbf{z}_{+}$and $\mathbf{z}_{-}$vectors; when this angle is small, the nonlinear coupling between the waves is further reduced, lengthening the nonlinear times. Perez \& Chandran (2013) found evidence for such alignments in models with long correlations times, but we find no evidence for such alignments in our simulations. Therefore, we omit the dependence on $\theta$ in the above definition of the nonlinear times. Figure 5 shows the nonlinear times $t_{\mathrm{nl}, \pm}$ (red and green curves) for the "smooth" model discussed in Section 3. The black curve shows the wave travel time $t_{0}(r)$, i.e., the time for an outwardpropagating wave to travel from the coronal base to a specific radial distance $r$. Note that the nonlinear time for the dominant outward waves is comparable to the wave travel time, $t_{\mathrm{nl},+} \sim t_{0}$. Therefore, the dominant waves do not have time to efficiently develop a turbulent spectrum before they escape into the region $r>20 R_{\odot}$. This explains why the power spectrum for these waves deviates significantly from a power law, see Figure 3(a). In contrast, the nonlinear time for the minority waves is much smaller than $t_{0}(r)$, so the turbulence is well developed for the minority waves.

In the present work we find that equation (7) significantly overestimates the dissipation rate compared to the value $Q_{\text {tot }}(r)$ derived from our numerical simulations. For example, at $r=2 R_{\odot}$ in the "smooth" model equation (7) overestimates the actual dissipation rate by about a factor 100. One possible reason for the discrepancy might be that the present numerical modeling is somehow deficient and severely underestimates the actual dissipation rate. It is true that the spatial resolution of our RMHD simulations is not very high, and our assumption of a flux tube with rigid boundary is questionable. However, we do not believe these effects can cause the dissipation rate $Q_{\text {tot }}$ to be underestimated by such a large factor. Another possibility is that equation (7) may not be applicable to our simulation, perhaps because one or more of the assumptions behind the equation are not valid for our case. However, we have not been able to identify any reason why the equation would not be applicable; the arguments by Chandran et al. (2009) in favor of this expression would seem to be valid in our case. Therefore, it is unclear why equation (17) gives such a poor fit to the numerically computed dissipation rate.

We tried other formulae for $Q_{\text {phen }}$ in an attempt to obtain a better fit. Since the first term in equation (7) is much larger than the second term, let us assume that the first term is somehow reduced by a factor $\mathcal{E}_{+}<1$. Then equation (77) can be generalized as follows:

$$
Q_{\text {phen }}=\rho_{0} \frac{\mathcal{E}_{+} Z_{+}^{2} Z_{-}+Z_{-}^{2} Z_{+}}{4 \lambda_{\perp}} .
$$


The largest reduction is obtained when $\mathcal{E}_{+} \sim Z_{-} / Z_{+}$, so that the dominant and minority waves have approximately equal contributions to the energy dissipation rate (further reduction of $\mathcal{E}_{+}$would have only a minor effect). Assuming exact equality of the two contributions $\left(\mathcal{E}_{+}=Z_{-} / Z_{+}\right)$, we obtain

$$
Q_{\text {phen }}=\rho_{0} \frac{Z_{-}^{2} Z_{+}}{2 \lambda_{\perp}} .
$$

We used this expression to compute $Q_{\text {phen }}(r)$ for both the "smooth" model and the model with density fluctuations. The blue curve in Figure 2 (f) shows $Q_{\text {phen }} / \rho_{0}$ for the "smooth" model discussed in Section 3. Note that at low heights equation (9) still overestimates the numerically computed rate $Q_{\text {tot }}$ (solid back curve); for example, at $r=2 R_{\odot}$ the ratio $Q_{\text {phen }} / Q_{\text {tot }} \approx 4$. Although this is not a good fit, the ratio is much smaller than that obtained with equation (7), which predicts $Q_{\text {phen }} / Q_{\text {tot }} \approx 100$. For $r>6 R_{\odot}$ equation (9) underestimates the numerically computed rate, but when $Q_{\text {phen }}$ is compared with only the perpendicular contribution $Q_{\perp}$ the agreement with the numerical results is significantly improved. The blue curve in Figure 4 (f) shows the phenomenological rate $Q_{\text {phen }} / \rho_{0}^{\prime}$ for the model with density fluctuations (Section 4). In this case equation (9) overestimates $Q_{\text {tot }}(r)$ by a factor ranging from 7 to 20. We conclude that equation (9) provides a much better fit to the data than equation (7), but still shows significant discrepancies between $Q_{\text {phen }}$ and $Q_{\text {tot. }}$

It should be mentioned that equation (9) depends on the rms amplitudes of the dominant and minority waves, $Z_{ \pm}(r)$. In the present work we were able to derive these amplitudes from the numerical RMHD simulations. However, for modeling the solar wind in the manner

of Cranmer et al. (2007) and Chandran et al. (2011) it would be useful to obtain accurate approximations for $Z_{ \pm}(r)$, so that the modeling can be done without doing computationally intensive RMHD simulations. Developing such approximations for models with density fluctuations is not trivial, and is beyond the scope of the present project. However, we realize that without such approximations the above equations for $Q_{\text {phen }}$ are of limited use.

\section{Discussion and Conclusions}

In this paper we considered a simple, one-fluid model of the fast solar wind, and we neglected all details of the collisionless processes by which the waves are dissipated at small spatial scales. In reality the solar wind exhibits significant departures from thermal equilibrium: different particle species have different temperatures, and particle velocity distributions can deviate significantly from Maxwellian (e.g., Feldman \& Marsch 1997; Kohl et al. 2006). When Alfvén wave energy cascades to the proton gyro-radius scale $\rho_{p}$, some of the energy may be dissipated by linear and nonlinear damping at that scale $\left(k_{\perp} \rho_{p} \approx 1\right)$, and 
the remainder of the energy may cascade into the kinetic Alfvén wave regime $\left(k_{\perp} \rho_{p} \gg 1\right)$, where the damping mainly benefits the electrons (see Chandran et al. 2011, and references therein). Therefore, the partitioning of the energy between ions and electrons depends on the details of these linear and nonlinear processes at and below the proton gyro-radius scale. In this paper we assume that the total dissipation rate is insensitive to the details of the dissipation process.

In Section 3 we found that in the model with a smooth background atmosphere the reflection-driven turbulence does not provide enough heating to maintain the assumed temperature, $T_{0}(r)$. We explored other values of the model parameters, and found that if the temperature is reduced [by using $C_{0}=0.3$ in equation (D10)] , the wave pressure force becomes even more dominant, and the outflow velocity at $r=20 R_{\odot}$ increases beyond 1000 $\mathrm{km} \mathrm{s}^{-1}$, too high for a realistic model of the fast solar wind. On the other hand, if the temperature is raised $\left(C_{0}=0.4\right)$, the required heating rate $Q_{\mathrm{A}}(r)$ increases in the central part of the model $\left(2 R_{\odot}<r<10 R_{\odot}\right)$, and the wave action parameter $S_{\mathrm{A}}(r)$ is reduced by $90 \%$ over the height range of the model, which is also not realistic (see, however, Hahn et al. 2012). If the wave amplitude at the coronal base is reduced from $40.6 \mathrm{~km} \mathrm{~s}^{-1}$ to $29.5 \mathrm{~km} \mathrm{~s}^{-1}$, the wave action parameter even becomes negative, so there is not enough energy to heat the plasma at larger heights. In all three cases the dissipation rate $Q_{\text {tot }}(r)$ remains well below the heating rate $Q_{\mathrm{A}}(r)$ needed in the central part of the model. Hence, there does not appear to be a smooth background atmosphere for which the turbulence can provide enough heating.

In Section 4 we considered the effects of density fluctuations on the propagation and reflection of the Alfvén waves. Such fluctuations may be due to compressive waves in the solar wind (Kudoh \& Shibata 1999; Moriyasu et al. 2004; Matsumoto \& Shibata 2010). We found that density variations with an rms amplitude of $10 \%$ and correlation length 0.04 $R_{\odot}$ produce strong wave reflections that significantly enhance the amplitude of the minority waves, and thereby the wave dissipation rate. The time-averaged wave dissipation rate is approximately equal to the plasma heating rate needed to maintain the temperature of the background atmosphere, i.e., the model with density fluctuations is approximately in thermal equilibrium. This suggest that Alfvén wave turbulence can heat and accelerate the fast solar wind, provided the effects of density fluctuations on wave reflection are taken into account.

In Section 5 we compared our simulation results with predictions from "phenomenological" turbulence models, taking into account the strong imbalance between dominant and minority waves $\left(Z_{+} \gg Z_{-}\right)$. We found that the standard formula for the energy dissipation rate, equation (77), significantly overestimates the numerically computed rate for the model with a smooth background atmosphere. The reasons why this formula gives such a poor fit are not fully understood. We proposed a revised formula based on the assumption that the 
cascade rate for the dominant waves is significantly reduced. We found that this revised formula [equation (9)] provides a better fit to the numerically computed rate, although there are still significant discrepancies. Cranmer et al. (2007) and Chandran et al. (2011) used the standard formula to construct detailed models of the solar wind, neglecting the effects of density fluctuations. We suggest that these authors may have overestimated the wave heating rate.

The ratio $Z_{-} / Z_{+}$is about a factor 10 larger in the model with density fluctuations than in the smooth model, so this quantity could be an important indicator for the presence of density fluctuations. Bavassano et al. (2000) used Ulysses observations to determine the energy densities of outward- and inward-propagating waves as function of radial distance in the heliosphere. Combining their results with Helios observations and extrapolating back to $r=0.1 \mathrm{AU}$, they find $Z_{-} / Z_{+} \sim 0.1$, similar to the value in our model with density fluctuations [see Figure 4(c)]. However, the waves observed in the heliosphere have periods of about 1 hour, much longer than the periods of the waves simulated here. Therefore, these heliosphere observations do not provide strong constraints on the present modeling. Such long-period waves may be produced by an inverse cascade of wave energy to large perpendicular scales. Since we consider only a single, relatively narrow flux tube $\left(R_{\text {base }}=1\right.$ $\mathrm{Mm}$ ), and do not include interactions between neighboring flux tubes, such a cascade cannot be described with the present model.

Morton et al. (2015) presented observational evidence for inward-propagating Alfvén waves in coronal holes, based on Dopplergrams obtained with the CoMP instrument. According to Figure 3 of their paper, the ratio of power spectra for inward and outward waves with frequencies in the range $3-7 \mathrm{mHz}$ is about 0.4 , much larger than the value of about 0.02 predicted by the present models [ratio $\left(Z_{-} / Z_{+}\right)^{2}$ derived from the red and green curves in Figure 2(a) or Figure 4(c) for $\left.r \approx 1.05 R_{\odot}\right]$. In our model the level of minority waves near the coronal base is mainly determined by the rapid rise in Alfvén speed from about $700 \mathrm{~km} \mathrm{~s}^{-1}$ at the coronal base $\left(r=1.003 R_{\odot}\right)$ to about $2000 \mathrm{~km} \mathrm{~s}^{-1}$ at $r \approx 1.3 R_{\odot}$, which causes wave reflection. In contrast, the observations show a nearly constant wave propagation speed of about $400 \mathrm{~km} \mathrm{~s}^{-1}$, which should produce less reflection than predicted by our model. This suggests that the observed inward waves are mainly produced by small-scale density fluctuations, not by reflections due to the overall height dependence of the mean Alfvén speed $v_{A}(r)$. Further observations of longitudinal and transverse waves in coronal holes would be very useful in clarifying the origin of the inward waves, and for constraining the type of models developed in this paper.

Figure 4(c) shows large fluctuations in the rms value of the Elsasser variable $Z_{-}(r)$ for the minority waves, even though the simulation results have been averaged over the cross- 
section of the flux tube and over time. The large fluctuations are likely an artifact of our assumption that the density variations $\delta \rho(r)$ are static, independent of time. In reality the density is expected to fluctuate in space and time, and we speculate that including the effects of temporal variability will reduce the magnitude of the fluctuations in $Z_{-}(r)$, but will not affect the mean value of $Z_{-}$, which will still be enhanced compared to a model without density fluctuations. However, this hypothesis cannot be tested with the present RMHD model, which assumes a fixed background atmosphere.

The present modeling still neglects any variations in density over the cross-section of the flux tube. However, large density variations across field lines have been observed (e.g., Woo 1996; Spangler 2002; Ravmond et al. 2014; Tian et al. 2011; Threlfall et al. 2013; Liu et al. 2015), and such variations likely have a significant effect on the propagation of Alfvén waves. To simulate the effects of perpendicular density variations on the Alfvén waves will require full MHD modeling. Such modeling is also needed to account for the coupling between Alfvén waves and other types of MHD waves. Previous studies have shown that density variations in the perpendicular direction can drastically change the nature of the waves, and can lead to phase-mixing and resonant absorption of the waves (e.g., Hevvaerts \& Priest 1983; De Groof \& Goossens 2002; Goossens et al. 2011, 2012, 2013; Pascoe et al. 2012). Future modeling of the fast solar wind using full MHD simulations should take such effects into account.

We thank the referee for providing detailed comments that helped improve the paper. We are most grateful to Alex Voss from the School of Computer Science at the University of St. Andrews for his support with the computational work. We thank Steve Cranmer for his thorough reading of the manuscript and helpful comments. We also thanks Hui Tian for pointing out recent observations relevant to our work. We are grateful to Benjamin Chandran for providing more information about the modeling results of Perez \& Chandran (2013). This project was supported under contract NNM07AB07C from NASA to the Smithsonian Astrophysical Observatory (SAO) and SP02H1701R from LMSAL to SAO. This research has made use of NASA's Astrophysical Data System. 


\section{A. Reduced MHD Model for the Solar Wind}

The RMHD equations are a simplified version of the full MHD equations (2), (3), (4) and (5). Actually, RMHD involves several approximations: (1) the magnetic fluctuations associated with the waves are assumed to have a transverse length scale $\ell_{\perp}$ that is small compared to their parallel scale $\ell_{\|} ;(2)$ the amplitude of the magnetic fluctuations is assumed to be small compared to the background field, $\left|\mathbf{B}_{1}\right| \ll B_{0}$; (3) the velocity fluctuations are assumed to be small compared to the Alfvén speed, $\left|\mathbf{v}_{1}\right| \ll v_{A}$; (4) the plasma pressure and density are assumed to be equal to their background values, $p \approx p_{0}$ and $\rho \approx \rho_{0}$, i.e., we neglect the coupling of the Alfvén waves with compressive, slow- and fast-mode waves; (5) the background density and field strength are assumed to be constant over the cross-section of the flux tube. Then the MHD equations can be split into two sets of coupled equations, one for the background medium and another for the Alfvén waves. In paper I we presented a detailed derivation of the RMHD equations for the case where the effects of parallel flows on the waves can be neglected, $u_{0} \ll v_{A}$. However, this approximation is not valid for the solar wind because $u_{0}=v_{A}$ at the Alfvén critical point, which is located at $r \sim 10 R_{\odot}$ (Cranmer et al. 2007; Perez \& Chandran 2013). Therefore, we now consider the effect of $u_{0}$ on the dynamics of the waves.

As in paper I, the background magnetic field $\mathbf{B}_{0}(\mathbf{r})$ is assumed to be a potential field, $\nabla \times \mathbf{B}_{0}=0$, and is locally approximated as

$$
\mathbf{B}_{0}(x, y, r) \approx B_{0} \hat{\mathbf{r}}-\frac{1}{2} \frac{d B_{0}}{d r}(x \hat{\mathbf{x}}+y \hat{\mathbf{y}}),
$$

where $r$ is the coordinate along the flux tube axis, $x$ and $y$ are coordinates perpendicular to the axis, $B_{0}(r)$ is the field strength on axis, and $\hat{\mathbf{r}}, \hat{\mathbf{x}}$ and $\hat{\mathbf{y}}$ are unit vectors. In this paper the flux tube is assumed to be radially oriented, but this is not essential for the equations

described in this Appendix. The unit vector $\hat{\mathbf{B}}_{0}$ along the background field varies over the cross-section of the tube, and is given by

$$
\hat{\mathbf{B}}_{0}(x, y, r) \approx \hat{\mathbf{r}}-\frac{1}{2 H_{\mathrm{B}}}(x \hat{\mathbf{x}}+y \hat{\mathbf{y}}),
$$

where $H_{\mathrm{B}}(r) \equiv B_{0} /\left(d B_{0} / d r\right)$ is the length scale for variations of the background field $\left(H_{\mathrm{B}}<\right.$ $0)$. The radius $R(r)$ of the tube is assumed to be small compared to $\left|H_{\mathrm{B}}\right|$. The Alfvén waves cause perturbations of the magnetic field $\mathbf{B}(\mathbf{r}, t)$ inside the tube. The induction equation (5) can be written in the form:

$$
\frac{\partial \mathbf{A}}{\partial t}=\mathbf{v} \times \mathbf{B}+\nabla \phi+\mathbf{D}_{A},
$$

where $\mathbf{A}(\mathbf{r}, t)$ is the vector potential $(\mathbf{B} \equiv \nabla \times \mathbf{A}), \phi(\mathbf{r}, t)$ is a scalar potential, $\mathbf{v}(\mathbf{r}, t)$ is the plasma velocity, and $\mathbf{D}_{A}$ is a dissipative term. The velocity field is approximated as

$$
\mathbf{v}(\mathbf{r}, t) \approx u_{0} \hat{\mathbf{B}}_{0}+\nabla_{\perp} f \times \hat{\mathbf{B}}_{0},
$$


where $u_{0}(r)$ is the outflow velocity of the solar wind, $f(\mathbf{r}, t)$ is the stream function of the velocity perturbations, and $\nabla_{\perp}$ is the derivative perpendicular to the background field, $\nabla_{\perp} \equiv$ $\nabla-\hat{\mathbf{B}}_{0}\left(\hat{\mathbf{B}}_{0} \cdot \nabla\right)$. Following Strauss (1997), we assume that the first-order perturbation of the vector potential $\mathbf{A}_{1}$ is parallel to the background field:

$$
\mathbf{A}_{1}(\mathbf{r}, t) \approx h(\mathbf{r}, t) \mathbf{B}_{0}(\mathbf{r}),
$$

where $h(\mathbf{r}, t)$ is the magnetic flux function. Since $\nabla \times \mathbf{B}_{0}=0$, it follows that the perturbed magnetic field can be approximated as

$$
\mathbf{B}(\mathbf{r}, t) \approx \mathbf{B}_{0}+\nabla_{\perp} h \times \mathbf{B}_{0} .
$$

Therefore, the cross-product of $\mathbf{v}$ and $\mathbf{B}$ is given by

$$
\mathbf{v} \times \mathbf{B} \approx u_{0} B_{0} \nabla_{\perp} h-B_{0} \nabla_{\perp} f+B_{0}\left[\hat{\mathbf{B}}_{0} \cdot\left(\nabla_{\perp} f \times \nabla_{\perp} h\right)\right] \hat{\mathbf{B}}_{0},
$$

and inserting this into equation ( $(\overline{\mathrm{A} 3})$, we obtain for the parallel and perpendicular components of this equation:

$$
\begin{aligned}
\frac{\partial h}{\partial t} & \approx \frac{1}{B_{0}} \hat{\mathbf{B}}_{0} \cdot \nabla \phi+\hat{\mathbf{B}}_{0} \cdot\left(\nabla_{\perp} f \times \nabla_{\perp} h\right), \\
0 & \approx u_{0} B_{0} \nabla_{\perp} h-B_{0} \nabla_{\perp} f+\nabla_{\perp} \phi .
\end{aligned}
$$

The latter can be integrated over $x$ and $y$ to yield an expression for $\phi$, and inserting this expression into equation (A8) yields

$$
\frac{\partial h}{\partial t}=\hat{\mathbf{B}}_{0} \cdot \nabla\left(f-u_{0} h\right)+\frac{f-u_{0} h}{H_{\mathrm{B}}}+[f, h]+D_{h},
$$

where $D_{h}$ is a dissipative term. Here the bracket operator is defined by

$$
[a, b] \equiv \frac{\partial a}{\partial x} \frac{\partial b}{\partial y}-\frac{\partial a}{\partial y} \frac{\partial b}{\partial x}
$$

where $x$ and $y$ are the coordinates perpendicular to the flux tube axis. All nonlinearities of the RMHD model are contained within such bracket terms.

A similar analysis can be applied to the equation of motion (3). The perpendicular component of this equation yields

$$
\left(\frac{d \mathbf{v}}{d t}\right)_{\perp}=\frac{u_{0}}{R} \hat{\mathbf{B}}_{0} \cdot \nabla\left(R \mathbf{v}_{1}\right)+\frac{\partial \mathbf{v}_{1}}{\partial t}+\mathbf{v}_{1} \cdot \nabla \mathbf{v}_{1}
$$

where we used equation (A2), and we assumed flux conservation ( $B_{0} R^{2}=$ constant). By taking the curl of equation (A12), we obtain the following vorticity equation:

$$
\frac{\partial \omega}{\partial t}=-u_{0}\left(\hat{\mathbf{B}}_{0} \cdot \nabla \omega-\frac{\omega}{H_{\mathrm{B}}}\right)-[\omega, f]+v_{A}^{2}\left\{\hat{\mathbf{B}}_{0} \cdot \nabla \alpha+[\alpha, h]\right\}+D_{\omega}
$$


where $\alpha(\mathbf{r}, t) \equiv-\nabla_{\perp}^{2} h$ is the magnetic torsion parameter, $\omega(\mathbf{r}, t)$ is the parallel component of vorticity:

$$
\omega(\mathbf{r}, t) \equiv \hat{\mathbf{B}}_{0} \cdot \nabla \times \mathbf{v}_{1} \approx-\nabla_{\perp}^{2} f
$$

and $D_{\omega}$ is a dissipative term. In deriving equation (A13) we neglected terms of higher order in $\epsilon\left(\equiv \ell_{\perp} / \ell_{\|}\right)$, and we used equation ( $\left.\underline{\mathrm{A} 2}\right)$ to compute the $x$ - and $y$-derivatives of $\hat{\mathbf{B}}_{0}$. The first term in equation (A13) describes the torque due to the expansion of the plasma in the parallel flow $u_{0}$. For a detailed derivation of the other terms, see paper I. The RMHD approximations greatly simplify the MHD equations, reducing them to two coupled equations (A10) and (A13) for two scalar quantities, $h(\mathbf{r}, t)$ and $f(\mathbf{r}, t)$. The key feature of the RMHD equations is that they retain the nonlinear terms responsible for the development of Alfvén wave turbulence. Note that the outflow velocity $u_{0}(r)$ only affects the linear terms in the equations.

\section{B. Numerical Methods}

In this paper we consider Alfvén waves propagating along a thin flux tube with circular cross-section. The radius $R(r)$ of the cross-section increases with distance $r$ along the tube. For an arbitrary point within the tube, let $\tilde{r} \equiv \sqrt{x^{2}+y^{2}} \leq R(r)$ be the distance from the axis, and let $\varphi$ be the azimuth angle. Then the scalar functions can be written as $h(\xi, \varphi, r, t)$ and $f(\xi, \varphi, r, t)$, where $\xi \equiv \tilde{r} / R$ is the fractional distance from the flux tube axis, and derivatives along the background field can be written as partial derivatives $\partial / \partial r$ at constant $\xi$ and $\varphi$. We use a spectral method to describe the dependence of $h$ and $f$ on the perpendicular coordinates $\xi$ and $\varphi$. Specifically, we use a set of orthogonal basis functions $F_{k}(\xi, \varphi)$ that are eigenmodes of the $\nabla_{\perp}^{2}$ operator and also satisfy the side boundary conditions on the flux tube (see Appendix B of paper I). The modes are enumerated by an index $k$ $(k=1, \cdots, N)$ and have well-defined perpendicular wavenumbers $k_{\perp}=a_{k} / R$, where $a_{k}$ is a dimensionless wavenumber (given by the zeros of Bessel functions). For the simulations presented in this paper, the maximum dimensionless wavenumber $a_{\max }=30$, which requires $N=209$ modes.

The magnetic and velocity fluctuations are described by the mode amplitudes $h_{k}(r, t)$ and $f_{k}(r, t)$, respectively. The RMHD equations can then be written as

$$
\begin{aligned}
\frac{\partial h_{k}}{\partial t}= & \frac{\partial}{\partial r}\left(f_{k}-u_{0} h_{k}\right)+\frac{f_{k}-u_{0} h_{k}}{H_{\mathrm{B}}}+\frac{1}{R^{2}} \sum_{j=1}^{N} \sum_{i=1}^{N} M_{k j i} f_{j} h_{i} \\
& -\nu_{k} h_{k}+\beta D^{6} h_{k},
\end{aligned}
$$




$$
\begin{aligned}
\frac{\partial \omega_{k}}{\partial t}= & -u_{0}\left(\frac{\partial \omega_{k}}{\partial r}-\frac{\omega_{k}}{H_{\mathrm{B}}}\right)+v_{A}^{2} \frac{\partial \alpha_{k}}{\partial r}+\frac{1}{R^{2}} \sum_{j=1}^{N} \sum_{i=1}^{N} M_{k j i}\left(v_{A}^{2} \alpha_{j} h_{i}-\omega_{j} f_{i}\right) \\
& -\nu_{k} \omega_{k}+\beta D^{6} \omega_{k},
\end{aligned}
$$

where $\alpha_{k}=\left(a_{k} / R\right)^{2} h_{k}$ and $\omega_{k}=\left(a_{k} / R\right)^{2} f_{k}$ are the mode amplitudes for magnetic torsion and vorticity, and $M_{k j i}$ is an anti-symmetric matrix describing the nonlinear couplings between the various modes (see paper I). Here we added artificial damping terms involving the parameters $\nu_{k}$ and $\beta$. The damping rate $\nu_{k}$ depends on the dimensionless perpendicular wavenumber $a_{k}$ of the waves. Note that the same damping $\nu_{k}$ is applied to both the magneticand velocity fluctuations, so the magnetic Prandtl number $P r_{M}=1$. For low perpendicular wavenumbers $\left(a_{k} \leq 15\right)$ we set $\nu_{k}=0$, so that the outward propagating waves can travel to large height without any damping. For high wavenumbers $\left(15 \leq a_{k} \leq 30\right)$ the damping rate increases linearly with $a_{k}$, and reaches its maximum value $\nu_{\max }$ at $a_{k}=30$. The maximum rate is given by $\nu_{\max }(r, t)=70 \overline{v_{\text {rms }}}(r, t) / R(r)$, where $v_{\text {rms }}(r, t)$ is the rms velocity of the waves, and the bar denotes a running time average over a time interval of $2000 \mathrm{~s}$. The terms with $\beta$ in equations (B1) and (B2) involve the sixth power of the dimensionless derivative operator $D \equiv\left(u_{0}+v_{A}\right) \Delta t \partial / \partial r$. The purpose of these terms is to prevent the build-up of waves with high parallel wavenumbers that cannot be adequately resolved on the radial grid; we use $\beta=0.001 /(64 \Delta t)$. The terms with $\nu_{k}$ and $\beta$ represent the physical processes that cause wave dissipation and heating of the coronal plasma.

The RMHD equations can also be formulated in terms of Elsasser-like variables, $\omega_{ \pm} \equiv$ $\omega \mp v_{A} \alpha$, where $\omega_{+}$and $\omega_{-}$are the vorticities of the dominant and minority waves, respectively (e.g., Perez \& Chandran 2013). For our spectral decomposition of the wave patterns, the wave equations are

$$
\frac{\partial \omega_{ \pm, k}}{\partial t}=-\left(u_{0} \pm v_{A}\right) \frac{\partial \omega_{ \pm, k}}{\partial r}-\left[\frac{d v_{A}}{d r} \pm \frac{u_{0}}{2 H_{\rho}}\right] v_{A} \alpha_{k}+\frac{u_{0}}{H_{\mathrm{B}}} \omega_{k}+\beta D^{6} \omega_{ \pm, k}+\cdots
$$

where $\omega_{ \pm, k}(r, t) \equiv \omega_{k} \mp v_{A} \alpha_{k}$ are the vorticity amplitudes of the individual modes, $H_{\rho}(r) \equiv$ $\rho_{0} /\left(d \rho_{0} / d r\right)$ is the density scale height, and the dots indicate nonlinear and $\nu_{k}$-damping terms. The first term on the right-hand side of equation (B3) describes the effects of wave propagation. Note that in the region beyond the Alfvén critical point (where $u_{0}>v_{A}$ ) the "inward" waves are actually carried outward by the flow. The second and third terms affect the amplification of the waves as they propagate outward or inward, and also include the linear couplings between the two modes. Note that these couplings occur only between modes with the same transverse wave pattern (indicated by index $k$ ). It can be shown that equation (B3) is equivalent to equation (14) of Perez \& Chandran (2013).

The RMHD equations are solved numerically, using the finite-difference method for the radial derivatives. The radial grid $r_{n}$ has 10,892 grid points. The grid is chosen such 
that the outward-wave propagation time between neighboring grid points is constant, $\left(r_{n+1}-\right.$ $\left.r_{n}\right) /\left(u_{0}+v_{A}\right)_{n+1 / 2}=\Delta t$, independent of $n$, where $\Delta t=1 \mathrm{~s}$ is the time step of the simulation. For each time step, we first compute the change in $h_{k}$ and $\omega_{k}$ due to wave propagation and reflection, using equation (B3). The first term in this equation describes wave propagation, and its effect is evaluated using the method of characteristics:

$$
\begin{aligned}
& \omega_{+, k}\left(\tilde{x}_{n}, t+\Delta t\right) \approx \omega_{+, k}\left(\tilde{x}_{n}-\Delta t, t\right)=\omega_{+, k}\left(\tilde{x}_{n-1}, t\right), \\
& \omega_{-, k}\left(\tilde{x}_{n}, t+\Delta t\right) \approx \omega_{-, k}\left(\tilde{x}_{n}+\tilde{f}_{n} \Delta t, t\right)
\end{aligned}
$$

where $\tilde{x}_{n}$ denotes the (outward) wave travel time at position $r_{n}$, and $\tilde{f}_{n}$ is the ratio of inward and outward wave speeds:

$$
\tilde{f}_{n} \equiv \frac{v_{A}\left(r_{n}\right)-u_{0}\left(r_{n}\right)}{v_{A}\left(r_{n}\right)+u_{0}\left(r_{n}\right)} .
$$

Equation (B4) shows that the dominant, outward-propagating waves $\omega_{+, k}$ simply move from one grid point to the next; this allows such waves to travel to large height in the model without any distortion of their radial profiles. However, for the minority waves we must use interpolation, and we use a fourth-order interpolation scheme:

$$
\omega_{-, k}\left(\tilde{x}_{n}, t+\Delta t\right) \approx \omega_{-, k}\left(\tilde{x}_{n}, t\right)+a_{n} \tilde{f}_{n}+b_{n} \tilde{f}_{n}^{2}+c_{n} \tilde{f}_{n}^{3}+d_{n} \tilde{f}_{n}^{4},
$$

where the coefficients $a_{n}, b_{n}, c_{n}$ and $d_{n}$ are determined from the values of $\omega_{-, i}$ at grid points $i=n-2, \cdots, n+2$ (we omit the detailed expressions). Near the inner and outer boundaries of the model we use quadratic or linear interpolation instead. Then the effects of the other linear terms in equation (B33) are added, and the result is converted to $h_{k}$ and $\omega_{k}$. Finally, we compute the change in $h_{k}$ and $\omega_{k}$ due to the nonlinear and $\nu_{k}$-damping terms in equations (B1) and (B2). This is done by integrating these equations over the time interval $[t, t+\Delta t]$, using a fourth-order Runge-Kutta method. The latter uses a variable time step that is often much smaller than $\Delta t$.

\section{Energy Equations for Waves and Plasma}

We first consider the energy equation for the waves. Let $U_{\text {mag }}(r, t)$ be the magnetic energy density of the waves, $\left|\mathbf{B}_{1}\right|^{2} / 8 \pi$, averaged over the cross-section of the flux tube. Similarly, let $U_{\text {kin }}(r, t)$ be the kinetic energy density of the waves, $\frac{1}{2} \rho_{0}\left|\mathbf{v}_{1}\right|^{2}$, averaged over the cross-section. In our RMHD model, these energy densities can be written as sums over eigenmodes:

$$
U_{\mathrm{mag}}(r, t)=\frac{B_{0}^{2}}{8 \pi R^{2}} \sum_{k=1}^{N} a_{k}^{2} h_{k}^{2}
$$




$$
U_{\text {kin }}(r, t)=\frac{\rho_{0}}{2 R^{2}} \sum_{k=1}^{N} a_{k}^{2} f_{k}^{2} .
$$

Multiplying equation (B1) by $B_{0}^{2} /(4 \pi)\left(a_{k} / R\right)^{2} h_{k}$ and summing over $k$, we obtain an equation for the time derivative of $U_{\mathrm{mag}}$, and multiplying (B2) by $\rho_{0} f_{k}$ we obtain the time derivative of $U_{\text {kin }}$. Adding these two equations, we find that the nonlinear terms drop out of the equation:

$$
\frac{\partial U_{\mathrm{A}}}{\partial t}+B_{0} \frac{\partial}{\partial r}\left(\frac{F_{\mathrm{A}}+U_{\mathrm{A}} u_{0}}{B_{0}}\right)=-U_{\mathrm{mag}} \frac{d u_{0}}{d r}+U_{\mathrm{kin}} \frac{u_{0}}{H_{\mathrm{B}}}-Q_{\mathrm{tot}},
$$

where $U_{\mathrm{A}}(r, t) \equiv U_{\text {mag }}+U_{\text {kin }}$ is the total energy density of the waves, and $F_{\mathrm{A}}(r, t)$ is defined by

$$
F_{\mathrm{A}}(r, t) \equiv-\frac{B_{0}^{2}}{4 \pi R^{2}} \sum_{k=1}^{N} a_{k}^{2} h_{k} f_{k} .
$$

The total dissipation rate $Q_{\text {tot }}(r, t)$ has two contributions:

$$
Q_{\text {tot }}(r, t) \equiv Q_{\perp}+Q_{\|}
$$

where

$$
\begin{aligned}
Q_{\perp}(r, t) & =\frac{\rho_{0}}{R^{2}} \sum_{k=1}^{N} a_{k}^{2} \nu_{k}\left(f_{k}^{2}+v_{A}^{2} h_{k}^{2}\right), \\
Q_{\|}(r, t) & =\beta \frac{\rho_{0}}{R^{2}} \sum_{k=1}^{N} a_{k}^{2}\left[\left(D^{3} f_{k}\right)^{2}+v_{A}^{2}\left(D^{3} h_{k}\right)^{2}\right] .
\end{aligned}
$$

The terms with $\nu_{k}$ describe damping at high perpendicular wavenumber, while those with $\beta$ describe damping at high parallel wavenumber. In deriving equation (C3) we neglected the contributions of $\beta$ terms to the energy flux. Equation (C3) is valid for arbitrary non-WKB wave propagation.

We now consider the equations for the background atmosphere. These are obtained by averaging the MHD equations over the cross-section of the flux tube, and over time. Then the mass conservation equation (2) yields

$$
\rho_{0} u_{0} / B_{0}=\text { constant }
$$

and the equation of motion (3) yields

$$
\rho_{0} u_{0} \frac{d u_{0}}{d r}=-\frac{d p_{0}}{d r}+D_{\mathrm{wp}}-\rho_{0} \frac{G M_{\odot}}{r^{2}} .
$$

Here $p_{0}$ is the plasma pressure, and $D_{\text {wp }}(r)$ is the wave pressure force. For an ideal gas $p_{0}=c_{1} \rho_{0} T_{0}$, and assuming a helium abundance of $10 \%, c_{1}=2.3 k_{\mathrm{B}} /\left(1.4 m_{\mathrm{H}}\right)$, where $k_{\mathrm{B}}$ is the 
Boltzmann constant and $m_{\mathrm{H}}$ is the hydrogen mass. For non-WKB Alfvén waves, the wave pressure force is given by (Heinemann \& Olbert 1980; Cranmer \& van Ballegooijen 2005):

$$
D_{\mathrm{wp}}(r)=-\frac{d U_{\mathrm{mag}}}{d r}+\frac{U_{\mathrm{mag}}-U_{\mathrm{kin}}}{H_{\mathrm{B}}} .
$$

Here $U_{\text {mag }}(r)$ and $U_{\text {kin }}(r)$ are the time-averaged versions of the quantities given in equations (C1) and (C2). The heat equation (41) can be written as

$$
Q_{\mathrm{A}}=Q_{\mathrm{adv}}+Q_{\mathrm{rad}}+Q_{\mathrm{cond}},
$$

where $Q_{\mathrm{A}}(r)$ is the time-averaged heating rate; $Q_{\mathrm{adv}}(r)$ is the time average of the advection terms [left-hand side of equation (4)]; and $Q_{\text {rad }}(r)$ and $Q_{\text {cond }}(r)$ are the energy loss rates due to radiation and thermal conduction. These quantities are given by

$$
\begin{aligned}
Q_{\mathrm{adv}}(r) & =c_{1} \rho_{0} u_{0}\left(\frac{1}{\gamma-1} \frac{d T_{0}}{d r}-\frac{T_{0}}{\rho_{0}} \frac{d \rho_{0}}{d r}\right), \\
Q_{\mathrm{rad}}(r) & =n_{\mathrm{e}} n_{\mathrm{H}} \Lambda\left(T_{0}\right), \\
Q_{\text {cond }}(r) & =B_{0} \frac{d}{d r}\left(\frac{F_{\text {cond }}}{B_{0}}\right),
\end{aligned}
$$

where $n_{\mathrm{H}}(r)=\rho_{0} /\left(1.4 m_{\mathrm{H}}\right)$ is the hydrogen density, $n_{\mathrm{e}}(r)=1.2 n_{\mathrm{H}}$ is the electron density, and $\Lambda(T)$ is the radiative loss function (taken from Figure 1 in Cranmer et al. 2007). Following Cranmer et al., we use a "bridging law" for the parallel component of the thermal conductive flux:

$$
F_{\text {cond }}(r)=\frac{\nu_{\text {coll }} F_{\mathrm{SH}}+\nu_{\text {exp }} F_{\mathrm{FS}}}{\nu_{\text {coll }}+\nu_{\text {exp }}}
$$

where $F_{\mathrm{SH}}(r) \equiv-\kappa d T_{0} / d r$ is the classical Spitzer-Harm prescription for thermal conduction, and $F_{\mathrm{FS}}(r)=1.5 \alpha_{c} n_{\mathrm{e}} u_{0} k_{\mathrm{B}} T_{0}$ is free-streaming heat flux that applies in the collisionless limit (we use $\alpha_{c}=4$ ). Also, $\nu_{\text {coll }}(r)$ is the electron-electron collision frequency, and $\nu_{\exp }=u_{0} /\left|H_{\rho}\right|$ is the wind expansion rate. Note that the conductivity depends strongly on temperature, $\kappa \propto T_{0}^{5 / 2}$ (for details, see Cranmer et al. 2007).

Multiplying equation (C9) by $u_{0}$ and adding equation (C11), we obtain the energy equation for the plasma:

$$
B_{0} \frac{d}{d r}\left(\frac{F_{\text {plasma }}}{B_{0}}\right)=Q_{\mathrm{A}}-Q_{\mathrm{rad}}+u_{0} D_{\mathrm{wp}}
$$

where $F_{\text {plasma }}$ is the energy flux carried by the plasma:

$$
F_{\text {plasma }}(r)=\frac{1}{2} \rho_{0} u_{0}^{3}+\frac{\gamma}{\gamma-1} p_{0} u_{0}-\rho_{0} u_{0} \frac{G M_{\odot}}{r}+F_{\text {cond }} .
$$


The four terms on the right-hand side represent the kinetic energy flux of the wind, the enthalpy flux, the gravitational energy flux, and the conductive flux, respectively. The energy equation for the waves is obtained by time-averaging equation (C3) and rearranging terms:

$$
B_{0} \frac{d}{d r}\left(\frac{F_{\text {waves }}}{B_{0}}\right)=-u_{0} D_{\mathrm{wp}}-Q_{\mathrm{tot}},
$$

where $D_{\mathrm{wp}}(r)$ is given by equation (C10), and $F_{\text {waves }}$ is the energy flux carried by the Alfvén waves (Heinemann \& Olbert 1980; Cranmer \& van Ballegooijen 2005):

$$
F_{\text {waves }}(r) \equiv F_{\mathrm{A}}+\left(2 U_{\text {mag }}+U_{\text {kin }}\right) u_{0} .
$$

Here $F_{\mathrm{A}}(r)$ and $Q_{\mathrm{tot}}(r)$ are the time averages of the quantities defined in equations (C4) and (C5).

\section{Setting Up the Background Atmosphere}

Three-dimensional RMHD modeling for a thin flux tube requires that we first set up a one-dimensional model for the background atmosphere inside the tube, i.e., a model for the magnetic field strength $B_{0}(r)$, density $\rho_{0}(r)$ and outflow velocity $u_{0}(r)$ as functions of position along the flux tube. We require that this model satisfy not only the mass conservation equation (C8) but also the equation of motion (C9). The latter includes the wave pressure force $D_{\mathrm{wp}}(r)$, which plays an important role in producing the fast solar wind emanating from coronal holes.

Following Cranmer et al. (2007), we approximate the wave pressure force by assuming that the dominant waves are much stronger than the minority waves, $\left|\omega_{+}\right| \gg\left|\omega_{-}\right|$. Then the mode amplitudes for velocity and magnetic field are highly correlated, $f_{k} \approx-v_{A} h_{k}$, and using this expression in equations (C2) and (C4) we find

$$
\begin{aligned}
U_{\text {kin }} & \approx U_{\text {mag }} \approx \frac{1}{2} U_{\mathrm{A}}, \\
F_{\mathrm{A}} & \approx 2 v_{A} U_{\mathrm{mag}} \approx v_{A} U_{\mathrm{A}} .
\end{aligned}
$$

Inserting these approximations into the time-averaged version of the wave energy equation (C3) yields the so-called wave action equation:

$$
\frac{d S_{\mathrm{A}}}{d r}=-\left(1+M_{A}\right) \frac{Q_{\mathrm{A}}}{B_{0}},
$$

where $S_{\mathrm{A}}(r)$ is the wave action per unit magnetic flux within the tube:

$$
S_{\mathrm{A}}(r) \equiv \frac{\left(u_{0}+v_{A}\right)^{2} U_{\mathrm{A}}}{B_{0} v_{A}}=\left(1+M_{A}\right)^{2} \frac{U_{\mathrm{A}}}{\sqrt{4 \pi \rho_{0}}}
$$


and $M_{A}(r) \equiv u_{0} / v_{A}$ is the Alfvén Mach number. Here we use $M_{A} \propto \rho_{0}^{-1 / 2}$, which follows from equation (C8). Inserting (D1) into equation (C10) yields $D_{\mathrm{wp}} \approx-\frac{1}{2} d U_{\mathrm{A}} / d r$, and the derivative in this expression can be computed from equations (D4) and (D3). This yields the following expression for the wave pressure force:

$$
D_{\mathrm{wp}} \approx-W_{U} \frac{d \rho_{0}}{d r}+\rho_{0} W_{Q},
$$

where $W_{U}$ and $W_{Q}$ are defined by

$$
\begin{aligned}
W_{U}(r) & \equiv \frac{U_{\mathrm{A}}}{4 \rho_{0}}\left(\frac{1+3 M_{A}}{1+M_{A}}\right), \\
W_{Q}(r) & \equiv \frac{Q_{\mathrm{A}}}{2 \rho_{0}\left(u_{0}+v_{A}\right)} .
\end{aligned}
$$

Inserting expression (D5) into equation (C9) and using mass conservation to eliminate the density, we find the so-called wind equation:

$$
\left(u_{0}-\frac{c_{1} T_{0}+W_{U}}{u_{0}}\right) \frac{d u_{0}}{d r}=-\frac{c_{1}}{B_{0}} \frac{d}{d r}\left(B_{0} T_{0}\right)-\frac{W_{U}}{B_{0}} \frac{d B_{0}}{d r}+W_{Q}-\frac{G M_{\odot}}{r^{2}},
$$

consistent with equation (58) of Cranmer et al. (2007). For the models considered in this paper, the temperature $T_{0}(r)$ is a known function of position, see equation (D10) below. Assuming the functions $W_{U}(r)$ and $W_{Q}(r)$ are also known, we can solve equation (D8) in a standard way: first find the position of the critical point $r_{\mathrm{c}}$ where the right-hand side of equation (D8) vanishes; then integrate equation (D8) upward and downward in height, starting from points just above and below the critical point, respectively. This yields the outflow velocity $u_{0}(r)$ at all heights. The density $\rho_{0}(r)$ can then be computed by using mass flux conservation and the boundary condition on density at the coronal base.

Since the functions $W_{U}(r)$ and $W_{Q}(r)$ are not known a priori, we must determine them iteratively. In each iteration we treat these quantities as known functions, and we solve the wind equation in the standard way (in the first iteration we set $W_{U}=W_{Q}=0$ ). This yields new or updated values for the outflow velocity $u_{0}(r)$ and density $\rho_{0}(r)$ as described above. We then compute the energy loss rates $Q_{\text {adv }}(r), Q_{\text {rad }}(r)$ and $Q_{\text {cond }}(r)$, and using equation (C11) we obtain an improved estimate for the heating rate $Q_{\mathrm{A}}(r)$ needed to sustain the background atmosphere. Next, we integrate equation (D3) from the base upward. This yields the wave action parameter $S_{\mathrm{A}}(r)$, from which we can determine the wave energy density $U_{\mathrm{A}}(r)$. Finally, we recompute $W_{U}(r)$ and $W_{Q}(r)$ from equations (D6) and (D7), and we repeat the iterative process, until the changes in $W_{U}$ and $W_{Q}$ become sufficiently small.

The temperature $T_{0}(r)$ must be specified in such a way that a critical point can always be found. For a proper critical point to exist, the function $F(r)$ on the right-hand side of 
equation (D8) must have a root, and the slope of the function at the root must be positive, $(d F / d r)_{\mathrm{c}}>0$. In particular, the first term in $F(r)$ related to temperature must be positive, and must decrease with $r$ at a rate which is less than that of the gravity term, $G M_{\odot} / r^{2}$. To ensure that this condition is satisfied, we specify not the temperature itself but rather the first term in $F(r)$ :

$$
-\frac{c_{1}}{B_{0}} \frac{d}{d r}\left(B_{0} T_{0}\right)=C_{0} \frac{G M_{\odot}}{R_{\odot}^{2}}\left(\frac{r}{R_{\odot}}\right)^{-m-1}\left[1-C_{1}\left(\frac{r}{R_{\odot}}\right)^{-k}\right],
$$

where $C_{0}$ and $C_{1}$ are dimensionless constants. For the first term on the right-hand side of equation (D9) to decreases more slowly than the gravity term in the wind equation, we require that the exponent $m<1$. Inserting equation (1) into (D9), we find for the temperature

$$
T_{0}(r)=\frac{G M_{\odot}}{c_{1} R_{\odot}} \frac{C_{0}}{B_{0}(r)} \sum_{n=1}^{5} B_{n}\left[\frac{1}{2 n+m}\left(\frac{r}{R_{\odot}}\right)^{-2 n-m}-\frac{C_{1}}{2 n+m+k}\left(\frac{r}{R_{\odot}}\right)^{-2 n-m-k}\right] .
$$

For the temperature to decrease with $r$ at large height, we require $m>0$. In the present paper we use $C_{0}=0.35, C_{1}=2, m=0.3$ and $k=8$. 


\section{REFERENCES}

Alazraki, G., \& Couturier, P. 1971, A\&A, 13, 380

Asgari-Targhi, M., \& van Ballegooijen, A.A. 2012, ApJ, 746, 81 (paper II)

Asgari-Targhi, M., van Ballegooijen, A.A., Cranmer, S.R., \& DeLuca, E.E. 2013, ApJ, 773, 111 (paper III)

Axford, I., \& McKenzie 1992, in Solar Wind Seven, 1

Bale, S. D., Kellogg, P. J., Mozer, F. S., Horbury, T. S., \& Reme, H. 2005, Phys. Rev. Letters, 94, 215002

Banerjee, D., Pérez-Suárez, D., \& Doyle, J. G. 2009, A\&A, 501, L15

Barnes, A. 1966, PhFl, 9, 1483

Bavassano, B., Pietropaolo, E., \& Bruno, R. 2000, J. Geophys. Res., 105, 15959

Belcher, J.W. 1971, ApJ, 168, 509

Belcher, J. W., \& Davis, L., Jr. 1971, JGR, 76, 3534

Bemporad, A., \& Abbo, L. 2012, ApJ, 751, 110

Beresnyak, A., \& Lazarian, A. 2008, ApJ, 682, 1070

Beresnyak, A., \& Lazarian, A. 2009a, ApJ, 702, 460

Beresnyak, A., \& Lazarian, A. 2009b, ApJ, 702, 1190

Berger, T. E., Loefdahl, M. G., Shine, R. S., \& Title, A. M. 1998, ApJ, 495, 973

Berger, T. E., \& Title, A. M. 1996, ApJ, 463, 365

Bhattacharjee, A., \& Ng, C. S. 2001, ApJ, 548, 318

Brandenburg, A. 2011, Astron. Nachr., 332, No. 1, 725

Buchlin, E., \& Velli, M. 2007, ApJ, 662, 701

Chandran, B. D. G., Dennis, T. J., Quataert, E., \& Bale, S. D. 2011, ApJ, 743, 197

Chandran, B. D. G., \& Hollweg, J. V. 2010, ApJ, 707, 1659 
Chandran, B. D. G., Quataert, E., Howes, G. G., Hollweg, J. V., \& Dorland, W. 2009, ApJ, 701,652

Chitta, L.P., van Ballegooijen, A.A., Rouppe van der Voort, L., DeLuca, E.E., \& Kariyappa, R. 2012, ApJ, 752, 48

Cho, J., Lazarian, A., \& Vishniac, E. T. 2002, ApJ, 564, 291

Coleman, P. J., Jr. 1968, ApJ, 153, 371

Coles, W. A., \& Harmon, J. K. 1989, ApJ, 337, 1023

Cranmer, S.R., Asgari-Targhi, M., Miralles, M. P., Raymond, J. C., Strachan, L., Tian, H., \& Woolsey, L. N. 2015, Phil. Trans. Royal Soc. A, 373, 20140148

Cranmer, S. R., \& van Ballegooijen, A. A. 2005, ApJS, 156, 265

Cranmer, S.R., van Ballegooijen, A.A., \& Edgar, R.J. 2007, ApJS, 171, 520

Cranmer, S. R., \& Woolsey, L. N. 2015, ApJ, 812, 71

DeVore, C. R., Sheeley, N. R., Jr., \& Boris, J. P. 1984, Sol. Phys., 92, 1

De Groof, A., \& Goossens, M. 2002, A\&A, 386, 691

De Pontieu, B., McIntosh, S. W., Carlsson, M., et al. 2007, Sci, 318, 1574

de Wijn, A. G., Stenflo, J. O., Solanki, S. K., \& Tsuneta, S. 2009, Space Sci. Rev., 144, 275

Dmitruk, P., \& Matthaeus, W. H. 2003, ApJ, 597, 1097

Dmitruk, P., Matthaeus, W. H., Milano, L. J., et al. 2002, ApJ, 575, 571

Dmitruk, P., Milano, L. J., \& Matthaeus, W. H. 2001, ApJ, 548, 482

Druckmüller, M., Habbal, S. R., \& Morgan, H. 2014, ApJ, 785, 14

Edwin, P. M., \& Roberts, B. 1983, Sol. Phys., 88, 179

Feldman, W. C., \& Marsch, E. 1997, in Cosmic Winds and the Heliosphere, ed. J. R. Jokipii, C. P. Sonett, \& M. S. Giampapa (Tucson: Univ. Arizona Press), 617

Goossens, M., Andries, J., \& Aschwanden, M. J. 2002, A\&A, 394, L39

Goossens, M., Andries, J., Soler, R., et al. 2012, ApJ, 753, 111 
Goossens, M., Erdélyi, R., Ruderman, M.S. 2011, Space Sci. Rev., 158, 289

Goossens, M., Van Doorsselaere, T., Soler, R., \& Verth, G. 2013, ApJ, 768, 191

Goldreich, P., \& Sridhar, S. 1995, ApJ, 438, 763

Goldreich, P., \& Sridhar, S. 1997, ApJ, 485, 680

Hahn, M., Landi, E., \& Savin, D. W. 2012, ApJ, 753, 36

Hartmann, L., \& MacGregor, K.B. 1980, ApJ, 242, 260

Heinemann, M., \& Olbert, S. 1980, JGR, 85, 1311

Heyvaerts, J., \& Priest, E.R. 1983, A\&A, 117, 220

Hollweg, J. V., \& Isenberg, P. A. 2007, JGR, 112, CiteID A08102

Hollweg, J. V. 1973, ApJ, 181, 547

Hollweg, J. V. 1986, J. Geophys. Res., 91, 4111

Hollweg, J. V., Jackson, S., \& Galloway, D. 1982, Sol. Phys., 75, 35

Hossain, M., Gray, P. C., Pontius, D. H., Jr., Matthaeus, W. H., \& Oughton, S. 1995, Phys. Fluids, 7, 2886

Iroshnikov, P. S. 1963, Astron. Zh., 40, 742 (English translation in Sov. Astron. 7, 566 [1964])

Jacques, S.A. 1977, ApJ, 215, 942

Jacques, S.A. 1978, ApJ, 226, 632

Kohl, J. L., Noci, G., Cranmer, S. R., \& Raymond, J. C. 2006, Astron. Astrophys. Rev., 13, 31

Kraichnan, R. H. 1965, Phys. Fluids, 8, 1385

Krishna Prasad, S., Banerjee, D., Van Doorsselaere, T., \& Singh, J. 2012, A\&A, 546, A50

Kudoh, T., \& Shibata, K. 1999, ApJ, 514, 493

Landi, E., \& Cranmer, S. R. 2009, ApJ, 691, 794

Liu, J., McIntosh, S. W., De Moortel, I., \& Wang, Y. 2015, ApJ, 806, article id. 273

Mallet, A., Schekochihin, A., \& Chandran, B. D. G. 2015, MNRAS, 446, L77 
Maron, J., \& Goldreich, P. 2001, ApJ, 554, 1175

Marsch, E., \& Tu, C.-Y. 1997, A\&A, 319, L17

Matsumoto, T., \& Shibata, K. 2010, ApJ, 710, 1857

Matthaeus, W. H., Goldstein, M. L., \& Roberts, D. A. 1990, JGR, 95, 20673

Matthaeus, W. H., Zank, G. P., Oughton, S., Mullan, D. J., \& Dmitruk, P. 1999, ApJ, 523, L93

McIntosh, S. W., De Pontieu, B., \& Tarbell, T. D. 2008, ApJ, 673, L219

Miyamoto, M., Imamura, T., Tokumaru, M., Ando, H., Isobe, H., Asai, A., et al. 2014, ApJ, 797,51

Moriyasu, S., Kudoh, T., Yokoyama, T., \& Shibata, K. 2004, ApJ, 601, L107

Morton, R. J., Tomczyk, S., \& Pinto, R. 2015, Nat. Comm., DOI: 10.1038/ncomms8813

Morton, R. J., Verth, G., Fedun, V., Shelyag, S., \& Erdélyi, R. 2013, ApJ, 768, 17

Muller, R., Roudier, T., Vigneau, J., \& Auffret, H. 1994, A\&A, 283, 232

Oughton, S., Matthaeus, W. H., Dmitruk, P., Milano, L. J., Zank, G. P., \& Mullan, D. J. 2001, ApJ, 551, 565

Parker, E. N. 1958, ApJ, 128, 664

Parker, E. N. 1960, ApJ, 132, 175

Parker, E. N. 1965, Space Sci. Rev., 4, 666

Pascoe, D. J., Hood, A. W., de Moortel, I., \& Wright, A. N. 2012, A\&A, 539, A37

Perez, J. C., \& Boldyrev, S. 2009, Phys. Rev. Letters, 102, 025003

Perez, J. C., \& Chandran, B. D. G. 2013, ApJ, 776, 124

Perez, J. C., Mason, J., Boldyrev, S., \& Cattaneo, F. 2012, Phys. Rev. X, 2, 041005

Raymond, J. C., McCauley, P. I., Cranmer, S. R., \& Downs, C. 2014, ApJ, 788, 152

Riley, P., Lionello, R., Linker, J. A., Mikic, Z., Luhmann, J., \& Wijaya, J. 2011, Sol. Phys., 274,361 
Shebalin, J. V., Matthaeus, W. H., \& Montgomery, D. 1983, J. Plasma Phys., 29, 525

Sheeley, N.R., Jr., Wang, Y.-M., \& DeVore, C. R. 1989, Sol. Phys., 124, 1

Singh, J., Hasan, S. S., Gupta, G. R., Nagaraju, K., \& Banerjee, D. 2011, Sol. Phys., 270, 213

Spangler, S. R. 2001, ApJ, 576, 997

Spruit, H. C. 1982, Sol. Phys., 75, 3

Stenflo, J. O. 1973, Sol. Phys., 32, 41

Strauss, H.R. 1976, Phys. Fluis, 19, 134

Strauss, H. R. 1997, J. Plasma Phys., 57, 83

Suzuki, T.K., \& Inutsuka, S.-I. 2005, ApJ, 632, L49

Suzuki, T.K., \& Inutsuka, S.-I. 2006, J. Geophys. Res., 111, A6, CiteID A06101

Threlfall, J., De Moortel, I., McIntosh, S. W., \& Bethge, C. 2013, A\&A, 556, A124

Tian, H., DeLuca, E. E., Cranmer, S. R., et al. 2014, Science, 346, 1255711

Tian, H., McIntosh, S. W., Habbal, S. R., He, J. 2011, ApJ, 736, 130

Title, A. M., Tarbell, T. D., \& Topka, K. P. 1987, ApJ, 317, 892

Tomczyk, S., \& McIntosh, S. W. 2009, ApJ, 697, 1384

Tomczyk, S., McIntosh, S. W., Keil, S. L., et al. 2007, Sci, 317, 1192

Tu, C., \& Marsch, E. 1995, Space Sci. Rev., 73, 1

Velli, M. 1993, A\&A, 270, 304

Velli, M., Grappin, R., \& Mangeney, A. 1989, Phys. Rev. Letters, 63, 1807

Verdini, A., Grappin, R., Pinto, R., \& Velli, M. 2012, ApJ, 750, L33

Verdini, A., \& Velli, M. 2007, ApJ, 662, 669

Verdini, A., Velli, M., \& Buchlin, E. 2009, ApJ, 700, L39

Verdini, A., Velli, M., Matthaeus, W. H., Oughton, S., \& Dmitruk, P. 2010, ApJ, 708, L116 
van Ballegooijen, A. A., Asgari-Targhi, M., Cranmer, S. R., \& DeLuca, E. E. 2011, ApJ, 736, article 3 (paper I)

Wang, Y.-M., Sheeley, N. R., Jr., Howard, R. A., et al. 1997, ApJ, 485, 875

Wilhelm, K., Marsch, E., Dwivedi, B. N., Hassler, D. M., Lemaire, Ph., Gabriel, A. H., \& Huber, M. C. E. 1998, ApJ, 500, 1023

Woo, R. 1996, Astrophys. \& Space Sci. 243, 97

Zhou, Y., \& Matthaeus, W. H. 1990, J. Geophys. Res., 95, 10291

Zirker, J. B. 1977, Coronal Holes and High Speed Wind Streams (Colorado Associated University Press: Boulder, Colorado) 

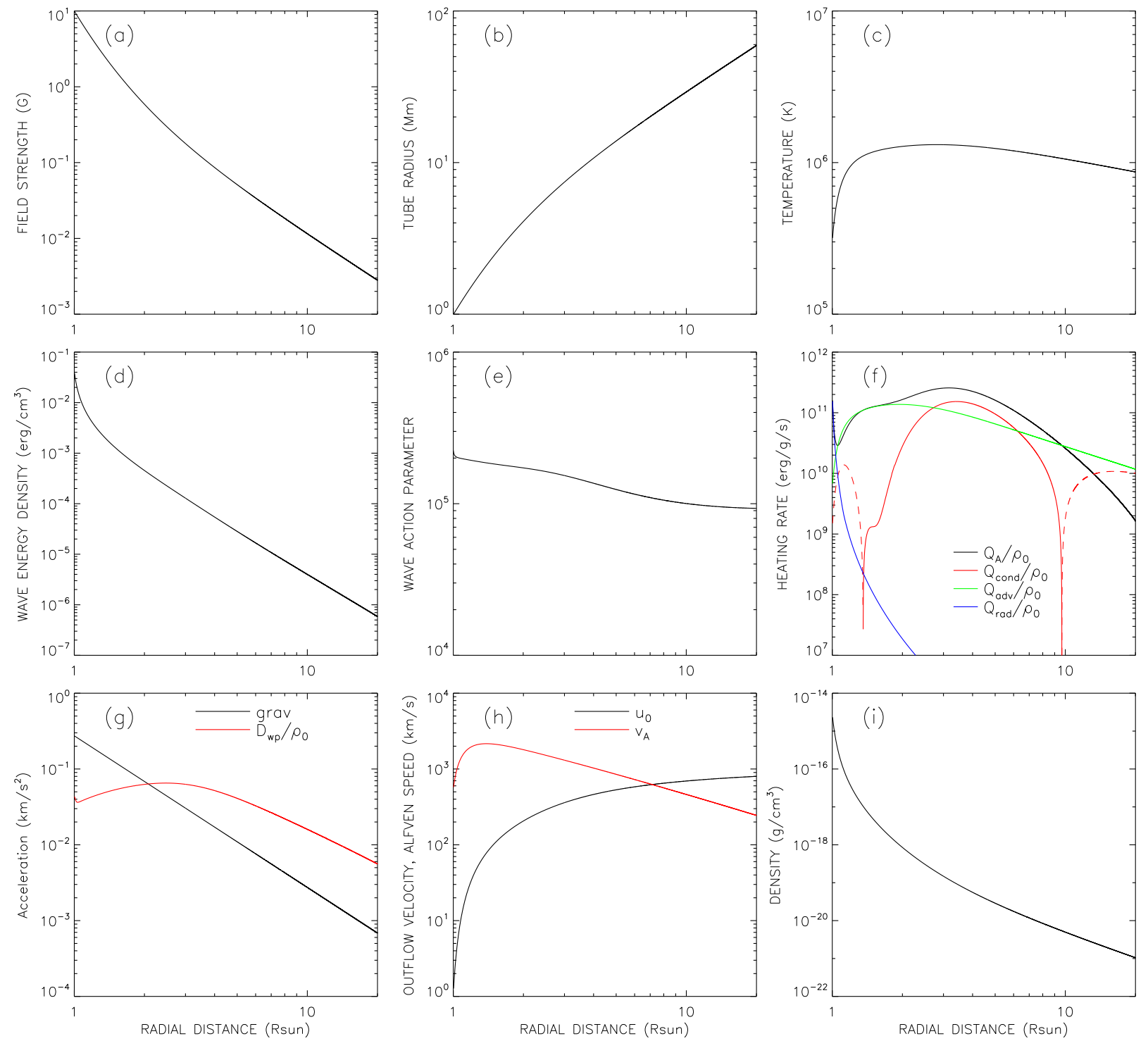

Fig. 1. - Radial dependence of various background quantities for a polar coronal hole. (a) Magnetic field strength. (b) Flux tube radius. (c) Temperature. (d) Wave energy density. (e) Wave action parameter. (f) Plasma heating rate due to wave dissipation (black curve), and energy loss rates due to thermal conduction (red curve), advection (green curve), and radiation (blue curve). (g) Outward acceleration due to wave pressure gradient (red curve), and inward acceleration due to gravity (black curve). (h) Outflow velocity (black curve) and Alfvén speed (red curve). (i) Mass density. 

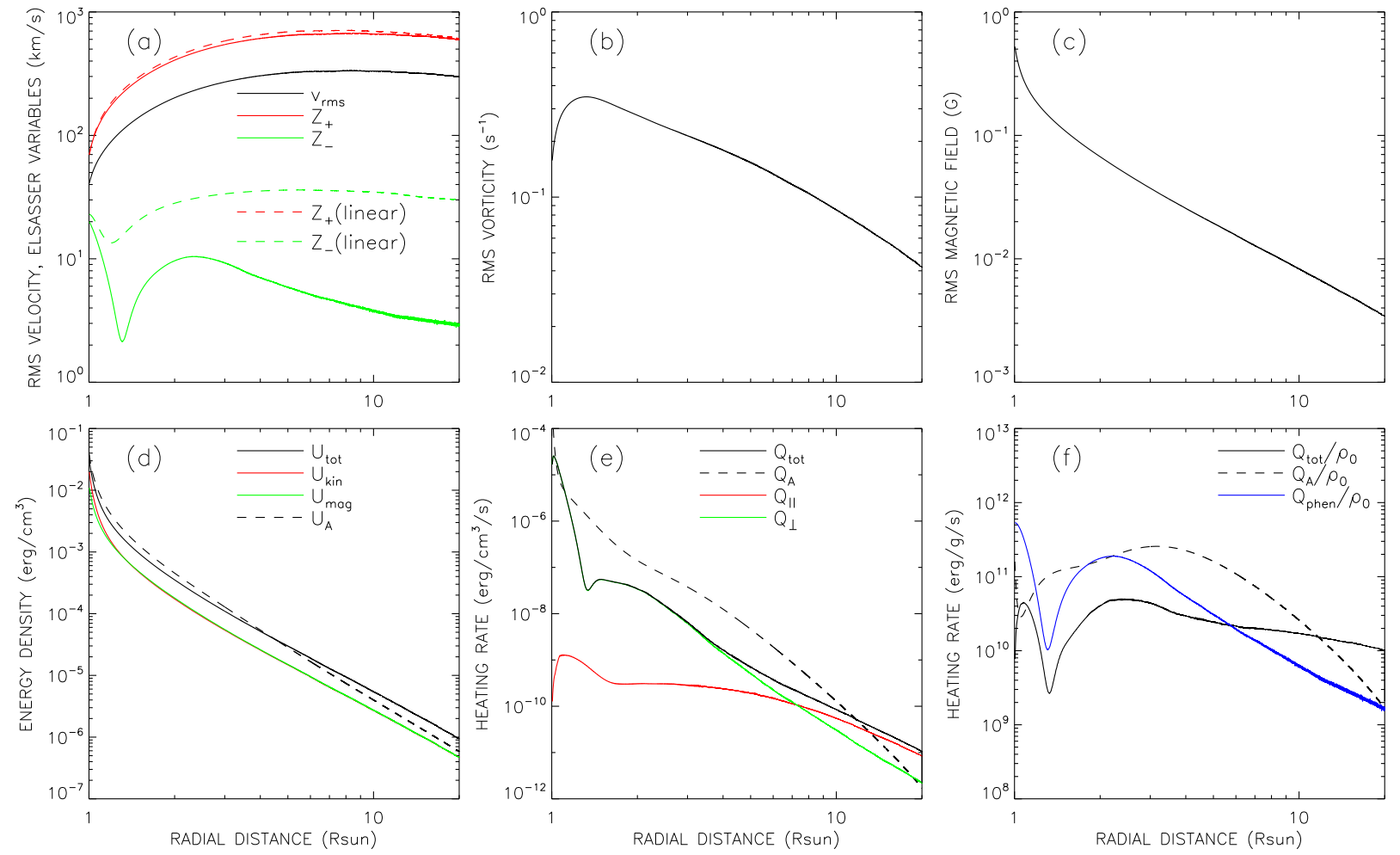

Fig. 2.- Radial dependence of various wave-related quantities for a polar coronal hole model, as derived from RMHD simulation. (a) Velocity amplitude of the waves (black curve), and Elsasser variables for dominant waves (red curve) and minority waves (green curve). The dashed red/green curves are for a model with the nonlinear terms switched off. (b) Amplitude of the parallel component of vorticity. (c) Amplitude of the fluctuating component of magnetic field. (d) Wave energy densities: total energy (black curve), kinetic energy (red curve), and magnetic energy (green curve). Also shown is the wave energy density assumed in setup of the background atmosphere (dashed curve). (e) Wave energy dissipation rates per unit volume: total wave dissipation rate $Q_{\text {tot }}$ (solid black curve), together with contributions from $Q_{\perp}$ (green curve) and $Q_{\|}$(red curve). Also shown is the plasma heating rate $Q_{\mathrm{A}}$ assumed in setup of the background atmosphere (dashed black curve). (f) Wave energy dissipation rates per unit mass: rate derived from turbulence simulation (solid black curve), rate assumed in the setup of background atmosphere (dashed curve), and rate predicted by the phenomenological model of equation (9) (blue curve). 

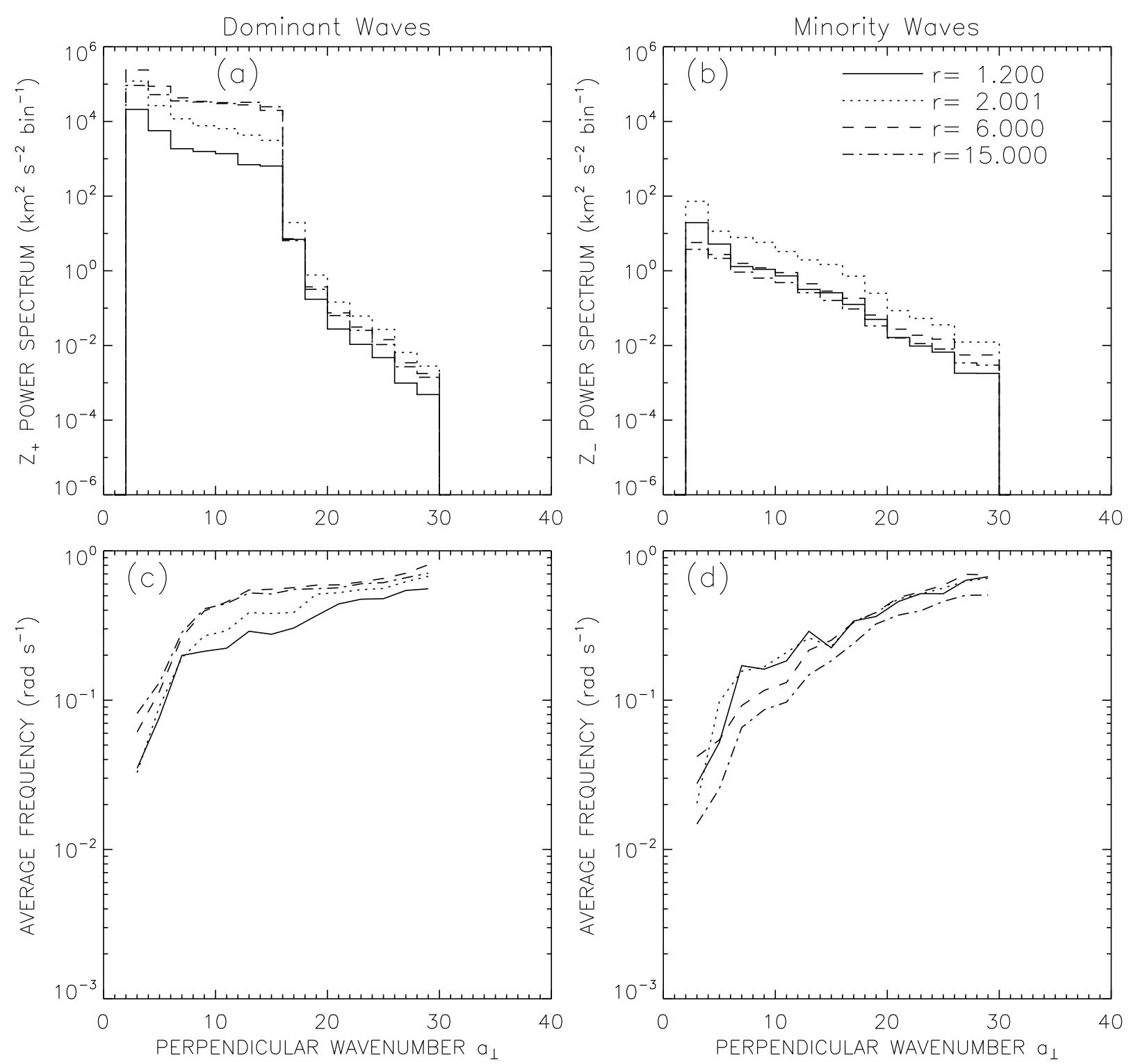

Fig. 3.- Spatial power spectra and wave frequencies as function of dimensionless perpendicular wavenumber $a_{\perp}$ for four different heights in the smooth model. (a) Power spectra for $Z_{+}$, the Elsasser variable for the dominant, outward propagating waves. The sharp drop at $a_{\perp}=15$ is due to the onset of $\nu_{k}$-damping at that wavenumber. (b) Power spectra for $Z_{-}$, the Elsasser variable for the minority waves, which also travel outward. (c) Average wave frequencies for dominant waves. (d) Average wave frequencies for minority waves. The different curves correspond to different heights: $r=1.2 R_{\odot}$ (solid), $r=2 R_{\odot}($ dotted), $r=6$ $R_{\odot}$ (dashed), $r=15 R_{\odot}$ (dash-dotted). 

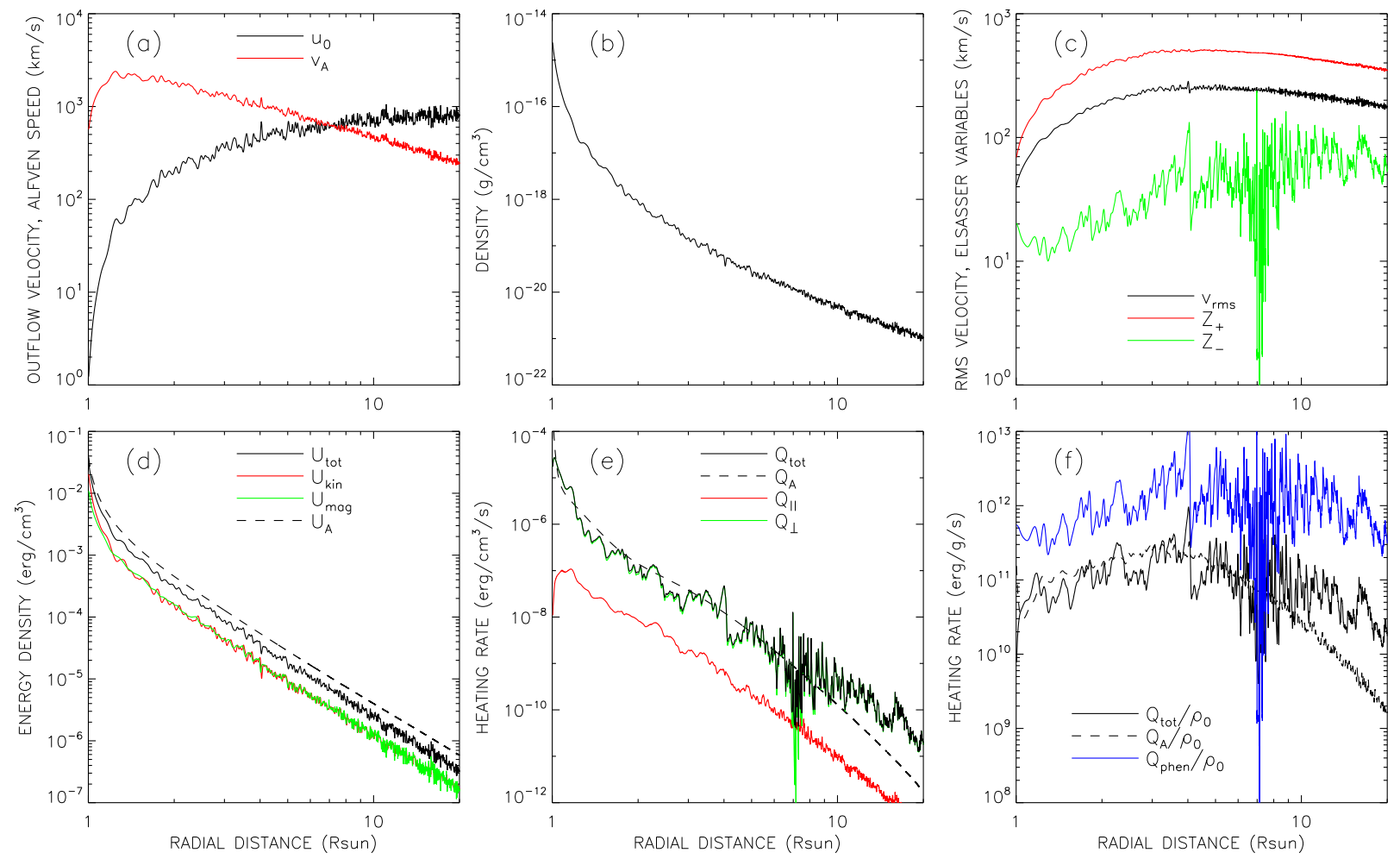

Fig. 4.- Radial dependence of various quantities for a polar coronal hole model with spatial variations in density along the flux tube. (a) Outflow velocity (black curve) and Alfvén speed (red curve). (b) Mass density. (c) Velocity amplitude of the waves (black curve), and Elsasser variables for the dominant waves (red curve) and minority waves (green curve). In this model the minority waves have both inward- and outward-propagating components. (d) Wave energy densities as derived from the RMHD simulation: total energy (black curve), kinetic energy (red curve), and magnetic energy (green curve). Also shown is the wave energy density assumed in setup of the background atmosphere (dashed curve). (e) Wave energy dissipation rates per unit volume: total wave dissipation rate $Q_{\text {tot }}$ (solid black curve), together with contributions from $Q_{\perp}$ (green curve) and $Q_{\|}$(red curve). Also shown is the plasma heating rate $Q_{\mathrm{A}}$ assumed in setup of the background atmosphere (dashed black curve). (f) Wave energy dissipation rates per unit mass: rate derived from turbulence simulation (solid black curve), rate assumed in the setup of background atmosphere (dashed curve), and rate predicted by a phenomenological model, equation (9) (blue curve). 


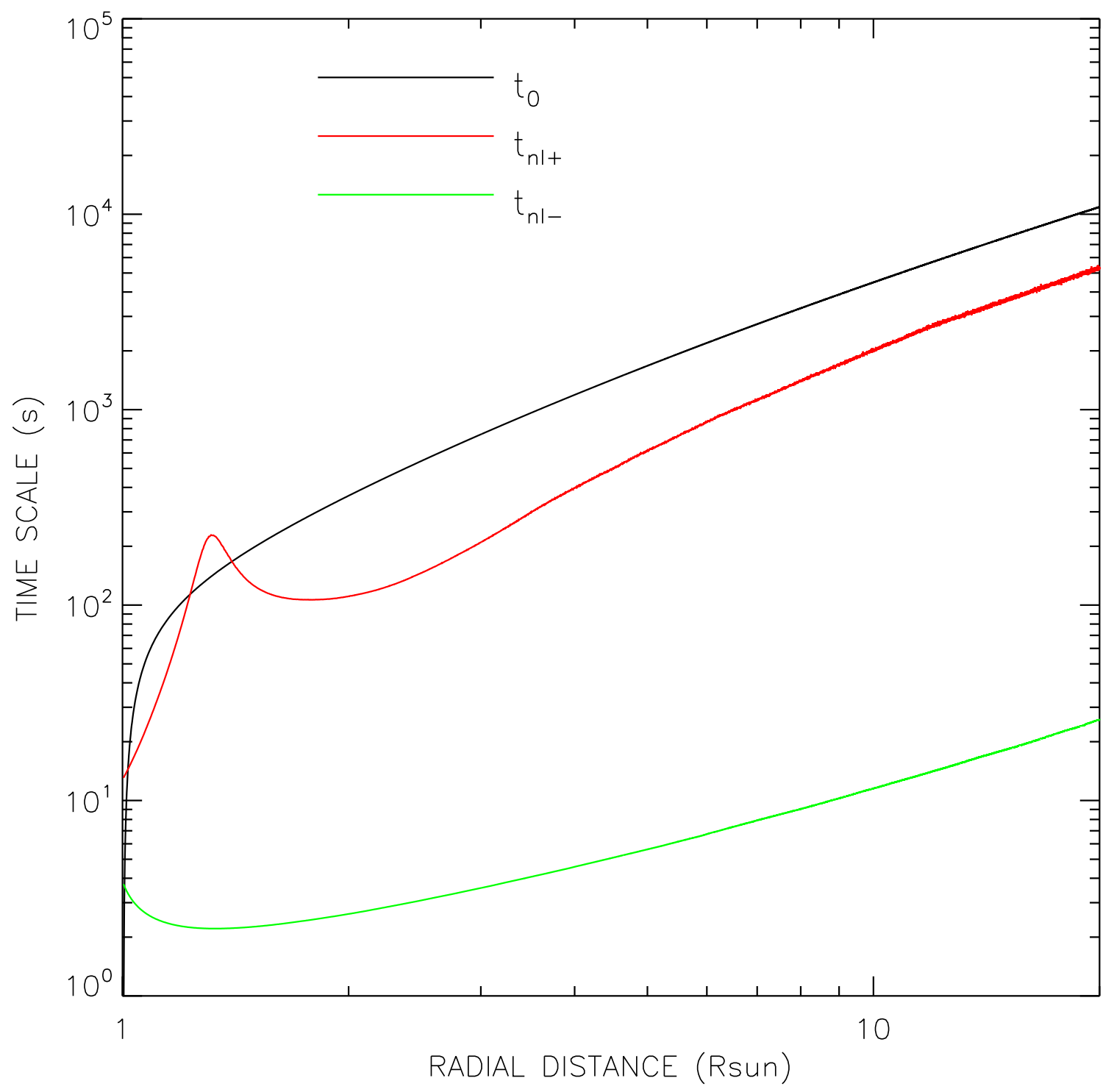

Fig. 5.- Various time scales as function of radial distance $r$ in the model with a smooth background atmosphere. The red and green curves show the nonlinear times $t_{\mathrm{nl}, \pm}(r)$ for the dominant and minority waves, respectively. The black curve shows the time $t_{0}(r)$ for an outward propagating wave to reach a certain height $r$. 